\title{
Packing Non-Identical Circles within a Rectangle with Open Length \\ Yaohua $\mathrm{He}^{\mathrm{a}, *}$, Yong Wu ${ }^{\mathrm{b}}$ \\ a Department of Industrial and Systems Engineering/Office of the Provost, National University of Singapore, Singapore 117576 \\ b Department of International Business and Asian Studies, Gold Coast Campus, Griffith University, QLD 4222 Australia
}

\begin{abstract}
Packing non-identical circles inside a rectangle witnesses a wide range of industrial applications. However, the non-convex constraints in this problem make it intractable using exact analytical approaches. Even via heuristic methods, the solution time for industrial-scale instances sometimes is too long to be acceptable. This article aims to challenge the existing methods for the benchmark instances. The most significant contributions of this work are: firstly, we proposed three types of packing positions for selection and used human intelligence to convert an arbitrary circle sequence into a feasible compact layout; secondly, diverse position selection criteria have been tested, and it is found that the criterion commonly used in the literature is not the best; thirdly, the traditional genetic algorithm is adapted with lower crossover rate but higher mutation rate particularly, and a minor-adjustment operator with the purpose of exploring the neighborhood of the current best solutions is introduced.
\end{abstract}

Keywords: cutting and packing; combinatorial optimization; heuristic; genetic algorithm

\section{Introduction}

In this study, we investigate the circle packing problem where a number of arbitrary-sized circles are to be packed into a two-dimensional rectangle with open length, i.e., one side of the rectangle is movable as long as it maintains parallel to the opposite side. The objective is to maximize the area utilization or equivalently, minimize the open length of the rectangle. The problem is known to be NP-complete [12]. Such a problem can find real-world applications in a wide range of industries, such as the textile, apparel, automobile, aerospace and chemical industries.

As pointed out by Castillo et al. [4], circle packing (mostly of identical circles) has been studied by many researchers in the "pure" mathematics area, but compared with the bin packing problem, it has obtained relatively less attention so far in the field of operations research, despite its extensive existing and potential applications.

Circle packing involves interesting modeling and computational challenges. In its general form, circle packing is a hard optimization problem that is intractable for exact analytical approaches. The “easiest” case of packing identical circles into a square or into a big circle are provably solved to

\footnotetext{
* Corresponding Author: Tel: +65-90438961, Fax: +65-67771434, E-mail: Yaohua.He001@gmail.com (Y. He)
} 
optimality only for a few instances (up to tens of circles), despite the great effort paid to variants of the problem in recent decades.

For the problem of packing non-identical circles into a rectangle, only several researchers have dedicated their efforts by proposing various models and heuristic methods. George et al. [6] proposed a mixed integer non-linear program for packing different circles into a rectangle of fixed dimensions. Stoyan and Yaskov $[16,17]$ used a mathematical model that searches for feasible local optima by combining a tree-search procedure and a reduced gradient method for packing non-identical circles into a rectangle with open length. Hifi and M'Hallah [10] came up with a constructive procedure and a genetic algorithm for the same problem. The constructive procedure utilizes a heuristic to select a set of favorite positions among all feasible positions according to the minimum distance between the packed circles and the current candidate circle; while the genetic algorithm is used to evolve a set of initial solutions. Hifi et al. [11] subsequently proposed a simulated annealing algorithm which starts by packing a set of specified circles into an open strip and tried to obtain a dense packing within the rectangle by exploiting the concept of the minimum distance between all selected circles. Huang et al. [12] presented two solution procedures, namely B1.0 and B1.5. B1.0 selects the next circle to place according to the maximum-hole degree rule and B1.5 improves the former with a self-look-ahead search strategy. Birgin et al. [3] coped with the same problem via a non-linear model. Akeb and Hifi [1] proposed an adaptive beam search on the basis of their previous work.

From the review of the existing methods, we can classify the solution techniques into three types. The first type consists of exact mathematical models, such as the studies by George et al. [6], Stoyan and Yaskov [16, 17], Birgin et al. [3], Yu and Zhang [19], Castillo et al. [4] and Kallrath [13]. Although the problem can be easily formulated as a general non-linear mathematical model, it is impossible to solve the model to global optimality just using the current local nonlinear solvers (like MINOS or other non-linear solvers) due to the well-known high-level non-convexity, as well as the exponentially increasing computational burden when the problem size grows. George et al. [6] formulated a mixed integer non-linear programming (MINLP) model for packing unequal circles into a rectangle. Stoyan and Yaskov [17] did not claim to compute the global optimum. The non-linear model proposed by Birgin et al. [3] has a number $O\left(n^{2}\right)$ of non-overlapping constraints. $\mathrm{Yu}$ and Zhang [19] formulated the problem of packing non-identical circles into a rectangle as a non-linear programming (NLP) problem, established the first order optimality conditions, and applied an augmented Lagrangian method to solve the problem. To demonstrate the performance of their proposed method, three instances were solved involving 33, 40 and 43 circles respectively. Castillo et al. [4] presented illustrative numerical results for various benchmark circle packing instances using three generic global solvers, LINGO, NMinimize and MathOptimizer Professional, to tackle the general models. Although they found "best known solutions" for some instances, there 
was no instance that has more than 35 circles $(N \leq 35)$. Kallrath [13] developed NLP and MINLP models describing the problem of cutting circles or polygons from rectangular design or stocked plates, and applied several solution techniques to solve this problem, where the solver Branch and Reduce Optimization Navigator (BARON) was called by GAMS. Numerical experiments indicated that the MINLP model can only solve small instances to global optimality.

The second type of approaches is deterministic enumeration based on certain greedy heuristics from human experience. One representative of this type is the work by Huang et al. [12], which can produce good results if enough time is allowed; however, the time-consumption associated with the high algorithm complexity (even with $O\left(n^{10}\right)$ ) is an issue if large-size instances are to be solved. Kubach et al. [14] put forward two greedy algorithms which are derived from the greedy algorithms proposed by Huang et al. [12], and parallelized these two algorithms using a shared memory master-slave approach to investigate initial configurations simultaneously.

The third type is stochastic search based on constructive heuristics or meta-heuristics. Hifi and his colleagues have been focusing on this problem over ten years and presented improving results in their publications [1, 2, 10, 11]. It has been noticed recently that Akeb et al. [2] came up with an augmented beam search-based algorithm, a modified version of the algorithm proposed by Akeb and Hifi [1]. Besides the beam search strategy, the modified algorithm was combined with a special binary search and a multi-start strategy. In order to improve the solution quality, a separate-beams strategy was incorporated in the tree-search procedure.

Although meta-heuristic methods cannot guarantee global optimality, they can provide near optimal solutions even for large-size problems within moderate or acceptable computational time, which consequently makes them currently the best choices for industrial-scale applications. Another advantage of meta-heuristics is that they can be more conveniently parallelized to exploit the power of high performance computation.

In this paper, based on our previous work on three-dimensional bin packing [9,18], we present a novel heuristic approach to construct a high-quality packing configuration for an arbitrary sequence of circles and an improved genetic algorithm to effectively evolve a set of initial solutions. Numerical experiment indicates that the proposed algorithm has faster solution speed than B1.0 and B1.5 by Huang et al. [12], and higher solution quality than the adaptive beam search by Akeb and Hifi [1] in solving small-size benchmarks. For the moderate- and large-size instances, we propose a decomposition approach which demonstrates to be more advantageous.

The rest of this paper is organized as follows: In Section 2 we define the problem to be solved; Section 3 introduces the procedure for converting a sequence of circles into a compact packing and Section 4 applies this procedure as the backbone for a tailored genetic algorithm; Section 5 conducts extensive numerical experiments and discusses the performance of the proposed method; Section 6 concludes this paper. 


\section{Problem Definition}

Assume that there are $N$ circles to be packed into a rectangle with unlimited length. The ultimate length of the rectangle is denoted by $L$ and the width of the container is denoted by $W$, while the center of circle $\pi_{i}$ is denoted by $\left(x_{i}, y_{i}\right)$ and the radius of circle $\pi_{i}$ is denoted by $r_{i}, i=1,2$, $3, \ldots, N$. The pairwise distance between the centers of circles $\pi_{i}$ and $\pi_{j}$, measured by the Euclidean distance, is denoted by $d_{i j}, d_{i j}=\sqrt{\left(x_{i}-x_{j}\right)^{2}+\left(y_{i}-y_{j}\right)^{2}}, i, j=1,2,3, \ldots, N$. Fig. 1 illustrates the rectangle, the coordinate system used and two circles.

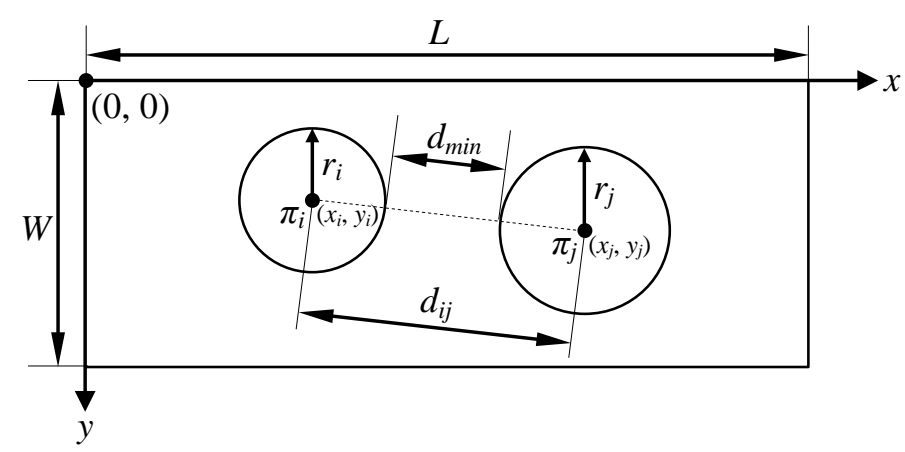

Fig. 1 Illustration of the rectangle, coordinate and two circles

The problem is to pack all the $N$ circles into the rectangle to minimize the length of the rectangle without overlap between any two circles. The non-linear model of the problem is formulated as follows [1, 4]:

$$
\begin{array}{ll}
\min & z=L ; \\
\text { s.t. } & \left(x_{i}-r_{i}\right) \geq 0, i=1,2,3, \ldots, N ; \\
& \left(y_{i}-r_{i}\right) \geq 0, i=1,2,3, \ldots, N ; \\
& \left(x_{i}+r_{i}\right) \leq L, i=1,2,3, \ldots, N ; \\
& \left(y_{i}+r_{i}\right) \leq W, i=1,2,3, \ldots, N ; \\
& \left(r_{i}+r_{j}\right) \leq d_{i j}, i, j=1,2,3, \ldots, N, i \neq j .
\end{array}
$$

The model involves three components: (i) the objective to be minimized; (ii) the constraints of the circles being placed within the rectangle; and (iii) the non-overlapping constraint between the circles. It can be observed that the formulation has $4 N$ linear constraints, and $N(N-1) / 2$ non-convex constraints. For the problem under investigation, $W$ is fixed, but $L$ is open for minimization.

\section{Packing an Arbitrary Sequence of Circles}

This section develops a procedure to convert an arbitrary sequence of circles into a compact packing. This procedure is subsequently used for decoding a chromosome into a phenotype solution in the genetic algorithm presented in Section 4. 


\subsection{Circle List and Position Lists}

Assume that $P=\left(\pi_{1}, \pi_{2}, \pi_{3}, \ldots, \pi_{N}\right)$ is a sequence of $N$ circles, $\pi_{i} \in\{1,2,3, \ldots, N\}, i=1,2,3, \ldots$, $N$. $P$ is a genotype solution, which is first changed into a circle list with $r_{i}, i=1,2,3, \ldots, N$, and then into a phenotype solution through placing each circle $\pi_{i}$ sequentially in the rectangle with the coordinate $\left(x_{i}, y_{i}\right)$ determined. Actually, the circle list with $\left(\pi_{i}, r_{i}, x_{i}, y_{i}\right), i=1,2,3, \ldots, N$, and other information, is a solution of packing configuration (see Table 6 in §5.1).

Imagine that in manual packing operation, one would try to place the current circle (to be packed) tangent to at least two items (either edges of the rectangle or circles already packed). This is one significant heuristic to determine the coordinate $\left(x_{i}, y_{i}\right)$ of the circle center of $\pi_{i}$. The other important heuristic is the criterion for selecting a position among the feasible candidate positions. Huang et al. [12] used the maximum hole degree $(\lambda)$ as the selection criterion, and Akeb and Hifi [1] exploited an equivalent criterion $\left(d_{\min }\right)$.

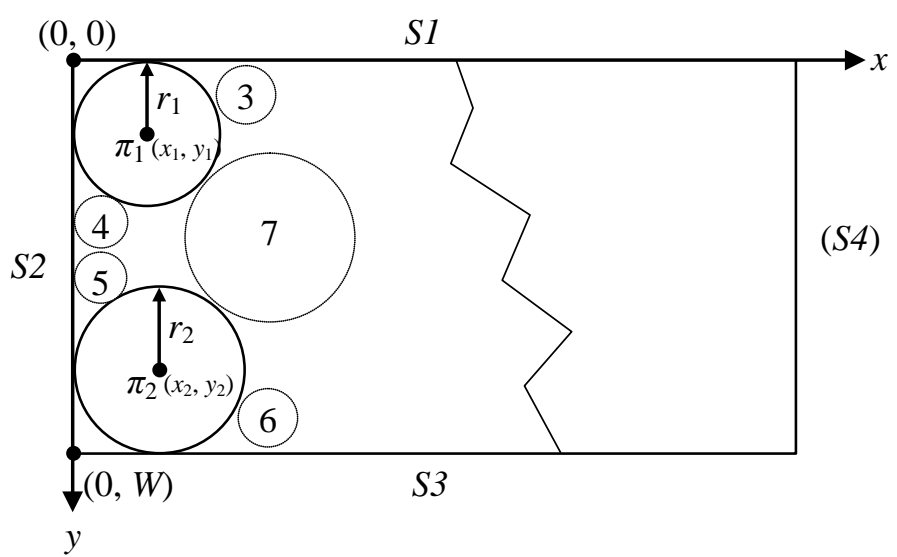

Fig. 2 Illustration of the candidate positions for selection

Fig. 2 illustrates an example of candidate positions for selection, where $S 1, S 2, S 3$ and $S 4$ denote the four edges of the rectangle. The open edge $S 4$ is not used as a reference for calculation since the packing is assumed to be carried out from left to right. When the rectangle is empty, it has two positions for selection, i.e. the two corners near points $(0,0)$ and $(0, W)$ respectively. Every time a circle is placed, at least two positions are generated for future assignment. For example, in Fig. 2, if circle $\pi_{1}$ is placed at the corner near point $(0,0)$, tangent to $S 1$ and $S 2$, positions 3 and 4 generated. Similarly, if circle $\pi_{2}$ is assigned to the corner near point $(0, W)$, tangent to $S 2$ and $S 3$, positions 5 and 6 are available. The positions directly generated from a circle packed belong to type one (I) positions which can be defined as follows.

Definition 1: A position of type one (I) is a "corner" formed by two adjacent items (an item can be either an edge or a circle); if a circle is assigned to this position, these three items are tangent to each other (three tangent points). Once a circle is packed, two type I positions on the open sides are added to position list I. At the end of packing $N$ circles, 2(N+1) type I positions are recorded in the list. 
As indicated in Fig. 2, if circle $\pi_{3}$ is small enough, one type I position among the four positions from 3 to 6 can be selected for packing. Apart from type I positions, we also have two other types of positions for selection. If circle $\pi_{3}$ is not so small (e.g., $r_{1}+r_{2}+r_{3}>W / 2$ in Fig. 2) as to assign to positions 4 and 5, a position of type two, position 7, needs to be considered. In this case, circle $\pi_{3}$ is tangent to circles $\pi_{1}$ and $\pi_{2}$, while $\pi_{1}$ and $\pi_{2}$ are not tangent to each other. Note that position 7 is taken into account to minimize the overall length of the packing; otherwise, type I positions 3 and 6 in Fig. 2 can still be used to place circle $\pi_{3}$. Type two (II) positions can be defined as below.

Definition 2: A position of type two (II) is a "corner" formed by two non-adjacent items; if a circle is assigned to this position, only two tangent points exist between these three items.

For programming convenience, we derive the definition of type three (III) positions from type II positions. If a type II position (e.g. the corner formed by $\pi_{3}$ and $\pi_{4}$ in Fig. 5) cannot accept a packing circle (e.g. $\pi_{5}$ ), then a position of type III is proposed (position 10 formed by $\pi_{3}$ and $S 1$ in Fig. 5). Type III is actually equivalent to type II, with regard to the two non-tangential items to constitute the placement corner.

Table 1. The information of a position (corner)

\begin{tabular}{|c|l|}
\hline Information & \multicolumn{1}{|c|}{ Specification } \\
\hline$\left(t m p \_x, t m p \_y\right)$ & Record the coordinate of the circle center of the circle being packed \\
\hline (index $1, i n d e x 2)$ & Record the values of the selection criteria, such as $x$ and $\lambda$, (one for MIN, the other for MAX) \\
\hline$\left(s_{1}, s_{2}, s_{3}\right)$ & Whether $S 1, S 2$ or $S 3$ forms the corner. If $S i$ do, set $s_{i}=1, i=1,2,3$ \\
\hline$\left(a j_{1}, x_{1}, y_{1}, r_{1}\right)$ & Circle $a d j_{1}$ to form the corner, the coordinate and radius of the circle \\
\hline$\left(a d j_{2}, x_{2}, y_{2}, r_{2}\right)$ & Circle $a d j_{2}$ to form the corner, the coordinate and radius of the circle \\
\hline$d_{\min }$ & The minimal distance between two items forming the corner. For I, $d_{\min }=0$; for II, III, $d_{\min }>0$ \\
\hline$a s \_f l a g$ & Assigned or not \\
\hline
\end{tabular}

To manage the positions for selection, we use three position lists (I, II and II) to store the information of the corresponding three types of positions. Each position in a position list at least includes the information shown in Table 1. If the current circle is assigned to a position, the coordinate of the circle center, (tmp_x,tmp_y), and the values of the selection criteria, (index1, index2), can be calculated from parameters of the two items forming the "corner" and the radius of the current circle. This calculation is case specific, using different formulas derived from respective cases.

Type I positions are directly generated through packing circles: every time a circle is packed, two type I positions are added to position list I as previously stated. But type II positions are added position list II during the feasibility check of type I positions in position list I for the current circle: if a type I position is "infeasible" (i.e., if the current circle is allocated at this type I position, it will intersect with other packed circle(s) or one edge among S1, S2 and S3), then a type II position is identified and put into position list II (if not existing yet). Type III positions are identified and added 
to position list III in a similar way. Therefore, there are a smaller number of positions in position lists II and III (usually less than $2 N$ at the end of packing $N$ circles). Since not all possible positions of types II and III are stored in the respective lists, the search time can be saved considerably.

It should be pointed out that consideration and utilization of positions of types II and III are necessary in order to obtain a high-quality packing configuration from a given sequence of circles; otherwise, the sequence may just yield a very poor packing, which will be illustrated later.

\subsection{Packing Procedure (Decoding Procedure)}

With the circle list and the position lists defined, the detailed flowchart of the packing procedure is given in Fig. 3. Through this procedure, $P=\left(\pi_{1}, \pi_{2}, \pi_{3}, \ldots, \pi_{N}\right)$ is first changed into a circle list and then the coordinate $\left(x_{i}, y_{i}\right)$ of each circle $\pi_{i}$ is determined. To begin with, circles $\pi_{1}$ and $\pi_{2}$ occupy the two corners near points $(0,0)$ and $(0, W)$ respectively (see Fig. 2$)$, and four type I positions are added to position list I. From circle $\pi_{3}$ onward, a proper position among position lists I, II and III is selected to pack each circle $\pi_{i}$ based on one selection criterion. Once a circle has been packed, the occupied position is deleted from the respective position list (is marked as “occupied”), and two new type I positions are added to position list I.

In Fig. 3, K1, K2 and $\mathbf{K} 3$ denote the numbers of all possible positions available for coming circles in position lists I, II and III respectively, and $n_{k 1}, n_{k 2}$ and $n_{k 3}$ denote the feasible positions for the current circle to be packed in position lists I, II and III respectively.

Fig. 4 illustrates two packing layouts for a sequence of circles in decreasing order of their radii (Table 2 presents the details of the solutions, by the position selection criterion "the minimum $x$ coordinate of circle $\pi_{i}$ ”, see the upcoming sub-section). In Fig. 4a, only type I positions are used for packing, but in Fig. 4b, the recorded positions of types II and III are also considered for selection. Apparently, the packing in Fig. 4b is more compact. It should be noted that, considering the recorded positions of types II and III does increase the computational complexity just from $O(2 n)$ to $O(4 n)$, but keeps the order of complexity unchanged, and eventually helps make the packing much more compact.

Table 2. The details of two packing solutions for a sequence of circles, where $W=30$

\begin{tabular}{|c|c|c|c|c|c|c|c|c|c|c|c|}
\hline & $i$ & 1 & 2 & 3 & 4 & 5 & 6 & 7 & 8 & 9 & 10 \\
\hline & $\pi_{i}$ & 1 & 2 & 3 & 4 & 5 & 6 & 7 & 8 & 9 & 10 \\
\hline & $r_{i}$ & 6.0 & 5.5 & 5.0 & 4.8 & 4.3 & 4.0 & 3.8 & 3.3 & 2.9 & 2.3 \\
\hline \multirow{2}{*}{ (a) } & $x_{i}$ & 6.000 & 5.500 & 15.988 & 11.630 & 16.159 & 20.381 & 24.243 & 3.300 & 20.977 & 22.770 \\
\cline { 2 - 12 } & $y_{i}$ & 6.000 & 24.500 & 25.000 & 16.223 & 4.300 & 17.145 & 3.800 & 14.899 & 9.650 & 27.700 \\
\hline \multirow{2}{*}{ (b) } & $x_{i}$ & 6.0000 & 5.5000 & 11.2097 & 15.7762 & 16.1409 & 20.0265 & 23.1826 & 23.1573 & 2.9000 & 22.4215 \\
\cline { 2 - 11 } & $y_{i}$ & 6.0000 & 24.5000 & 15.6881 & 25.2000 & 7.8031 & 17.4945 & 3.8000 & 10.9000 & 14.3427 & 27.7000 \\
\hline
\end{tabular}


Get a sequence $P=\left(\pi_{1}, \pi_{2}, \pi_{3}, \ldots, \pi_{N}\right)$;

Change $P$ into a circle list, and the coordinate

$\left(x_{i}, y_{i}\right)$ of each circle $\pi_{i}$ need to be determined;

Two corners of the empty rectangle are set as

two initial positions in position list I.

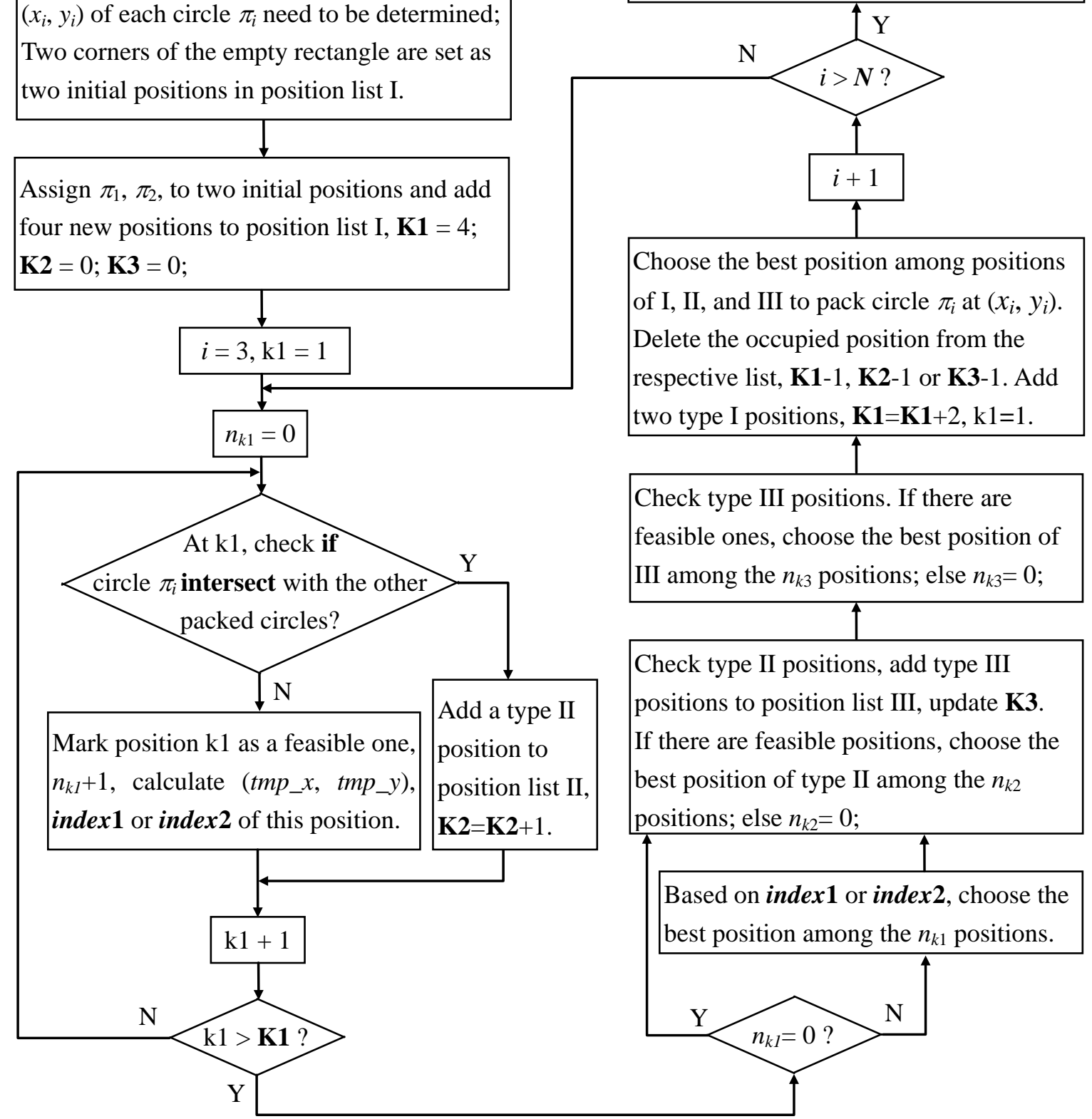

Fig. 3 Packing procedure

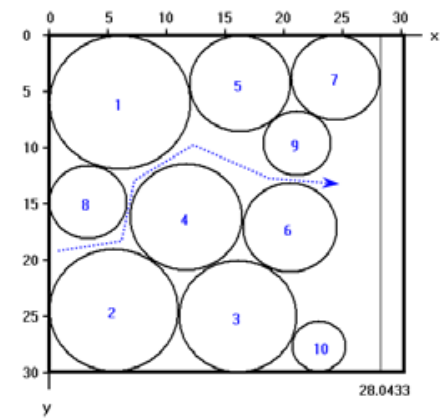

(a) Solely using type I positions

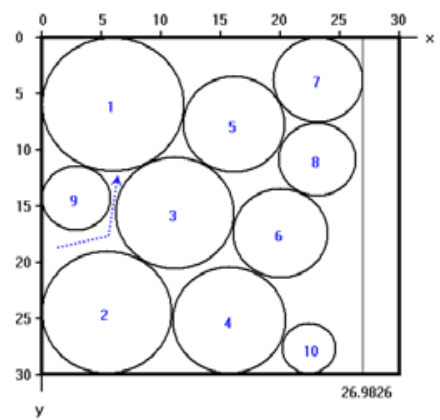

(b) Using positions of types I-III

Fig. 4 Comparison between solely using type I and using all types

Output the solution (i.e. the circle list with $\left.\left(x_{i}, y_{i}\right)\right)$, length $\boldsymbol{L}$ and the area utilization.

Choose the best position among positions , II, and III to pack circle $\pi_{i}$ at $\left(x_{i}, y_{i}\right)$ Delete the occupied position from the

Check type III positions. If there are III among the $n_{k 3}$ positions; else $n_{k 3}=0$;

Check type II positions, add type III If there are feasible positions, choose the

Based on index 1 or index 2 , choose the best position among the $n_{k 1}$ positions. 


\subsection{Criteria for Position Selection}

In order to pack an arbitrary sequence of circles into the rectangle from the left side (S1) to the right side (S4), the specific position selection for each circle is based on various criteria which concequently lead to different packing quality for the same circle sequence. Therefore, the use of an appropriate criterion is significant considering that a large set of sequences have to be searched by the algorithm. We consider five criteria in this work.

The first criterion considered is the minimum $x$ coordinate of circle $\pi_{i}$, i.e. $x_{i}$. That is, calculate the coordinates $\left(x_{i}, y_{i}\right)$ at all feasible positions, and select the position that lets circle $\pi_{i}$ have the minimum $x_{i}$. This criterion is denoted by $x$ in relevant tables.

The second criterion is the maximum hole degree $\lambda$ proposed by Huang et al. [12]:

$$
\lambda=1-d_{\text {min }} / r_{i},
$$

where $d_{\min }$ is the minimal distance from circle $\pi_{i}$ (whose radius is $r_{i}$ ) to other circles packed so far and edges of the rectangle (excluding the two items touching circle $\pi_{i}$ ) [12]. Akeb and Hifi [1] used an equivalent criterion, namely, selecting the position with the minimal $d_{\min }$ value. We denote this criterion as $\lambda$ and ignore $S 4$ since the length of the rectangle is variable.

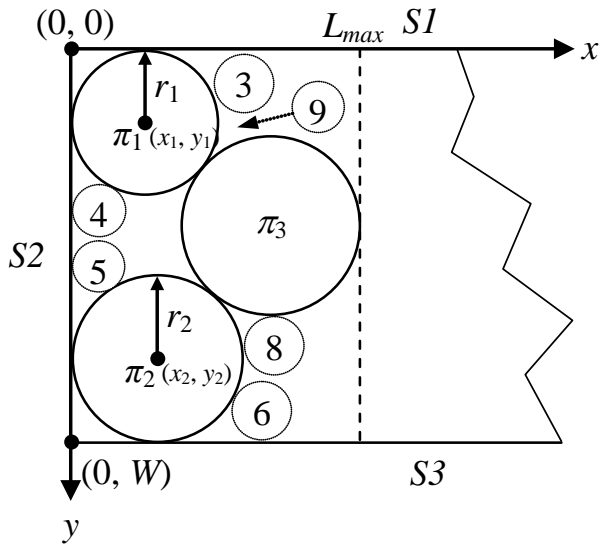

(a) Candidate positions for $\pi_{4}$

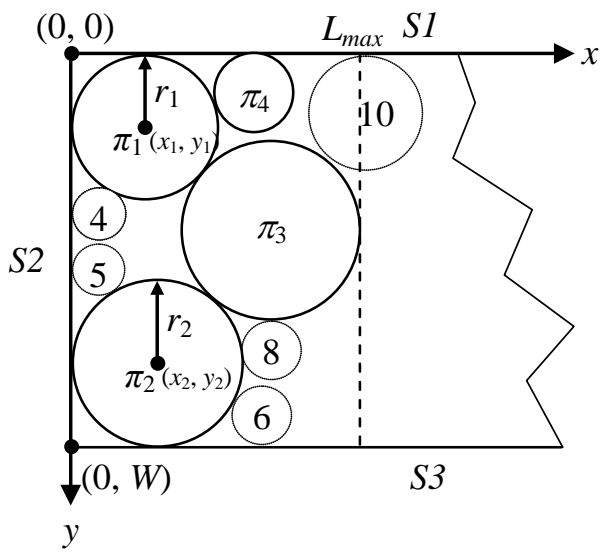

(c) Candidate positions for $\pi_{5}$

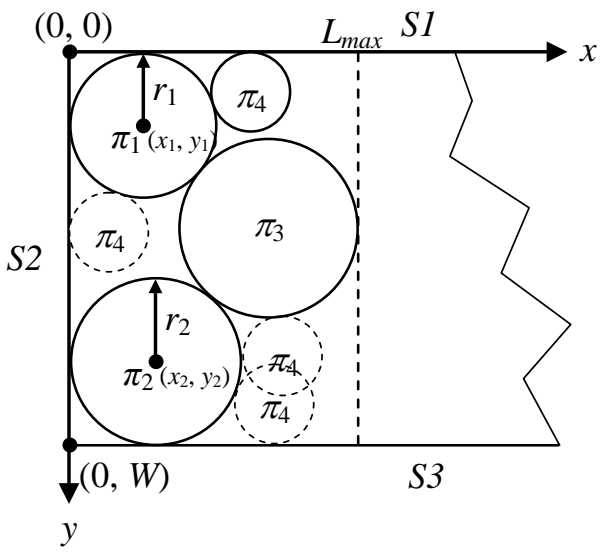

(b) $\pi_{4}$ assigned at 4 with minimum $d_{\min }$

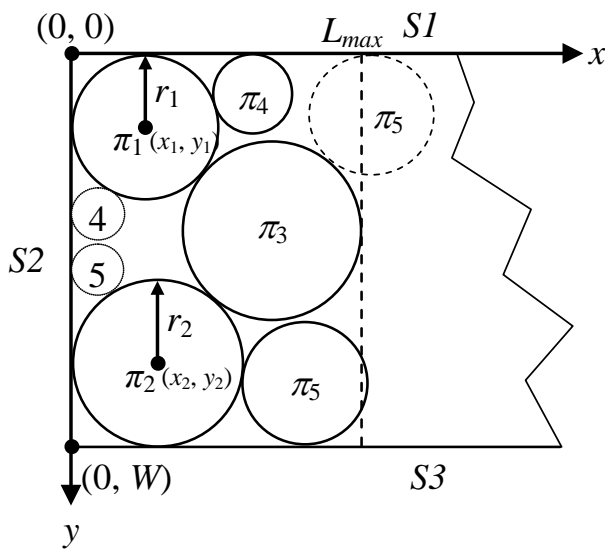

(d) $\pi_{5}$ assigned at 6 with minimum $x$ coordinate

Fig. 5 Packing circles using the third criterion $(\lambda$ and $x)$ 
The third selection criterion is to use the maximum $\lambda$ or the minimum $x$ coordinate according to whether the current circle can be packed within the current length $L_{\max }$ (see Fig. 5). If the circle can be packed within $L_{\max }$, the maximum $\lambda$ criterion is adopted; otherwise, the minimum $x$ coordinate criterion is accepted. The third criterion is denoted by $(\lambda$ and $x$ ).

Fig. 5 illustrates how the third criterion is applied. In Fig. 5a, $\pi_{1}, \pi_{2}$ and $\pi_{3}$ have been packed ( $\pi_{3}$ is packed at position 7, see Fig. 2), Positions 3, 4, 5, 6, 8 and 9 within $L_{\max }$ are the feasible candidate positions for the coming circle $\pi_{4}$. Since position 3 is with the maximum $\lambda$, it is chosen for $\pi_{4}$ (see Fig. 5b). In Fig. 5c, positions 6, 8 and 10 are three feasible ones for packing $\pi_{5}$, each of which lets $\pi_{5}$ exceed $L_{\text {max }}$. Position 6 enables $\pi_{5}$ have the minimum $x$ coordinate, thus it is selected (see Fig. 5d). Positions 4 and 5 are left for a more suitable circle later.

The fourth criterion considered is the maximum $\lambda / x$ and the fifth one is the minimum $\left(d_{\min }+x\right)$. From the experience in three-dimensional bin packing [9], the third criterion ( $\lambda$ and $x$ ), which distinguishes the situation inside the current length $L_{\max }$ from the one exceeding $L_{\max }$, should have good performance; but the real application in following computational tests is not like what we expected. Moreover, the commonly used $\lambda$ ( or $d_{\min }$ ) is not the best index to evaluate a position for packing a circle. The other criteria (e.g. $x$ and $d_{\min }+x$ ) lead to better solutions. However, the proper criterion may be instance-dependent.

\subsection{Heuristic of Decreasing Order and Exchange-Order Operations}

By using the packing procedure as shown in Fig. 3 and applying a position selection criterion, an arbitrary sequence of circles can be converted into a feasible packing configuration. In practice, heuristics are usually utilized for quick and better initial solutions, such as packing the circles in the decreasing order of their radii, and the exchange-order method mentioned in Akeb and Hifi [1], where the circles are partially re-arranged by specifying certain circles to be packed first in an iterative way. In this sub-section, we applied these two heuristics for the benchmark instances SY1 to SY6 initially used in Stoyan and Yaskov [17] with the position selection criteria and compared with the results by Akeb and Hifi [1] (see Tables 3 and 4). Fig. 6 shows the packing configurations of SY6 obtained from the decreasing order of circle radii.

From Table 3, it can be observed that, when packing in decreasing order of radii, our packing procedure with the first selection criterion obtains a better average objective value compared with OSGSP by Akeb and Hifi [1]. Especially for the two large-size instances, SY5 and SY6, we get better objective values. Fig. 6, compared with Fig. 4 in Akeb and Hifi [1], demonstrates the advantage of first selection criterion $(x)$ over the second one $\left(\lambda\right.$ or $\left.d_{\min }\right)$. In our procedure with the first selection criterion, whenever we pack a circle, the objective (i.e., the resulting length) is instantly considered, so that the remaining area within the end edge is small. 
Table 3. Packing in decreasing order of radii under different position selection criteria

\begin{tabular}{|c|c|c|c|c|c|c|c|c|}
\hline \#Inst & $N$ & $W$ & $L_{\text {OSGSP }}{ }^{*}$ & $L_{x}$ & $L_{\lambda}$ & $L_{(x \text { and } \lambda)}$ & $L_{\lambda / x}$ & \\
\hline SY1 & 30 & 9.5 & 18.4136 & 18.7153 & 20.5712 & 20.5712 & 20.5712 & 18.6787 \\
\hline SY2 & 20 & 8.5 & 16.8254 & 15.5980 & 16.1313 & 16.1313 & 16.1313 & 15.6069 \\
\hline SY3 & 25 & 9.0 & 15.2054 & 15.4437 & 15.9116 & 15.9116 & 15.6077 & 15.8420 \\
\hline SY4 & 35 & 11.0 & 24.9282 & 24.9504 & 25.2552 & 25.2577 & 25.0549 & 25.1710 \\
\hline SY5 & 100 & 15.0 & 38.4229 & 38.2607 & 38.7155 & 38.4580 & 38.4973 & 38.3406 \\
\hline SY6 & 100 & 19.0 & $\mathbf{3 9 . 7 8 3 6}$ & $\mathbf{3 8 . 9 2 7 6}$ & 46.7638 & 45.5040 & 98.9147 & 39.0156 \\
\hline mean & & & $\mathbf{2 5 . 5 9 6 5}$ & $\mathbf{2 5 . 3 1 6 0}$ & 27.2248 & 26.9723 & & 25.4425 \\
\hline
\end{tabular}

* From Akeb and Hifi [1]

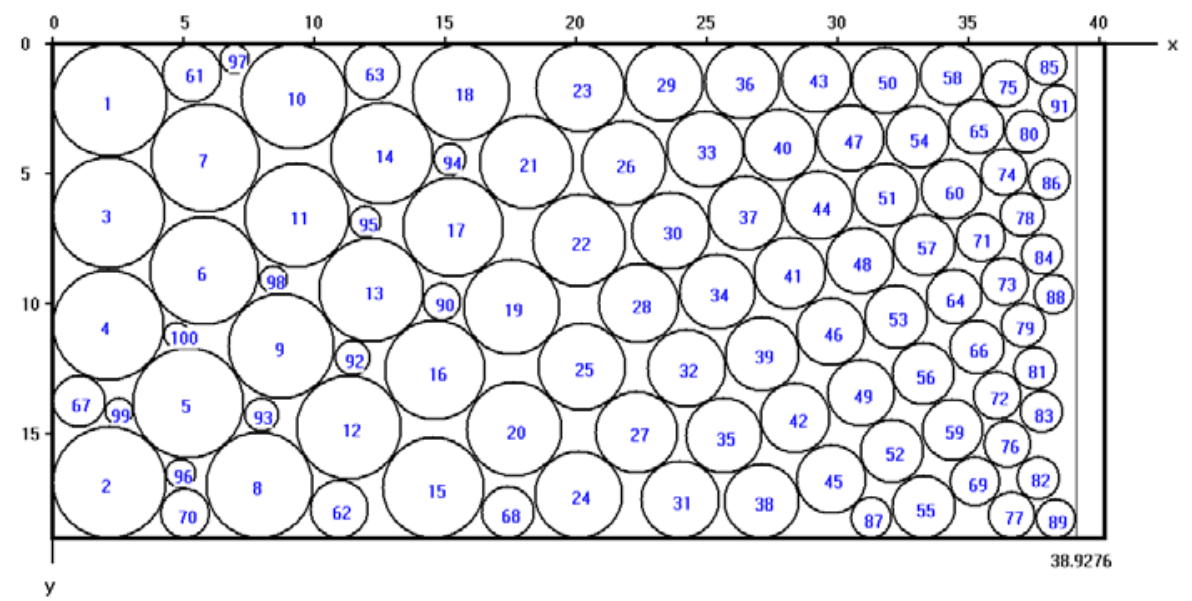

Fig. 6 A packing layout of SY6 from the decreasing order of radii $(L=38.9276)$

Table 4. Results from ExOrd1 under different position selection criteria

\begin{tabular}{|c|c|c|c|c|c|c|c|c|}
\hline \#Inst & $N$ & $W$ & $L_{\text {OSGSPa }}{ }^{*}$ & $L_{x}$ & $L_{\lambda}$ & $L_{(x \text { and } \lambda)}$ & $L_{\lambda / x}$ & \\
\hline SY1 & 30 & 9.5 & 18.1438 & 18.3096 & 18.6914 & 18.2328 & 18.3091 & 18.3096 \\
\hline SY2 & 20 & 8.5 & 14.8128 & 15.4148 & 15.5721 & 15.5721 & 15.5721 & 15.4709 \\
\hline SY3 & 25 & 9.0 & 15.1978 & 15.1138 & 15.5027 & 15.5027 & 15.5691 & 15.1704 \\
\hline SY4 & 35 & 11.0 & 24.4719 & 24.8923 & 24.9526 & 24.9526 & 25.0362 & 24.7786 \\
\hline SY5 & 100 & 15.0 & 37.2993 & 37.7895 & 38.5558 & 38.4436 & 38.4973 & 37.7687 \\
\hline SY6 & 100 & 19.0 & 38.0657 & 38.2349 & 39.4123 & 39.8717 & 39.3563 & 38.3728 \\
\hline mean & & & 24.6652 & 24.9591 & 25.4478 & 25.4293 & 25.3900 & 24.9785 \\
\hline
\end{tabular}

* From Akeb and Hifi [1]

Get the current best circle sequence from $\operatorname{ExOrd1},\left(\pi_{1}, \pi_{2}, \pi_{3}, \ldots, \pi_{\mathrm{N}}\right)$;

$i=2$;

while $(\mathrm{i}<N)$ do $\left\{1\right.$. Carry out the exchange-order: $\left(\pi_{i}, \pi_{i+1}, \pi_{1}, \ldots, \pi_{i-1}, \pi_{i+2}, \ldots, \pi_{N}\right)$;

2. Call the packing procedure with the new sequence;

3. $i++$.

\}

Find out the best sequence among the above $(\mathrm{N}-2)$ sequences;

If improved, update the current best circle sequence.

Fig. 7 Another exchange-order method (ExOrd2) 
We also propose another exchange-order method (simplified as ExOrd2), as displayed in Fig. 7, on top of the exchange-order method (simplified as ExOrd1) given by Akeb and Hifi [1]. Table 5 presents the results of SY1 to SY6 by ExOrd2 under diverse position selection criteria. Fig. 8 depicts the packing configuration of SY6 obtained from ExOrd2.

Table 5. Results from ExOrd2 under different position selection criteria

\begin{tabular}{|c|c|c|c|c|c|c|c|}
\hline \#Inst & $N$ & $W$ & $L_{x}$ & $L_{\lambda}$ & $L_{(x \text { and } \lambda)}$ & $L_{\lambda / x}$ & \\
\hline SY1 & 30 & 9.5 & 18.3096 & 18.3971 & 18.2328 & 18.3091 & 18.3096 \\
\hline SY2 & 20 & 8.5 & 15.4148 & 15.5721 & 15.5721 & 15.5721 & 15.4709 \\
\hline SY3 & 25 & 9.0 & 15.0135 & 15.4074 & 15.4070 & 15.5083 & 14.9955 \\
\hline SY4 & 35 & 11.0 & 24.8923 & 24.9526 & 24.9526 & 25.0362 & 24.7244 \\
\hline SY5 & 100 & 15.0 & 37.5753 & 38.3227 & 38.4436 & 38.3467 & 37.7687 \\
\hline SY6 & 100 & 19.0 & $\mathbf{3 7 . 8 6 4 7}$ & 39.3859 & 39.5856 & 38.8311 & 38.3632 \\
\hline mean & & & 24.8450 & 25.3396 & 25.3656 & 25.2673 & 24.9387 \\
\hline
\end{tabular}

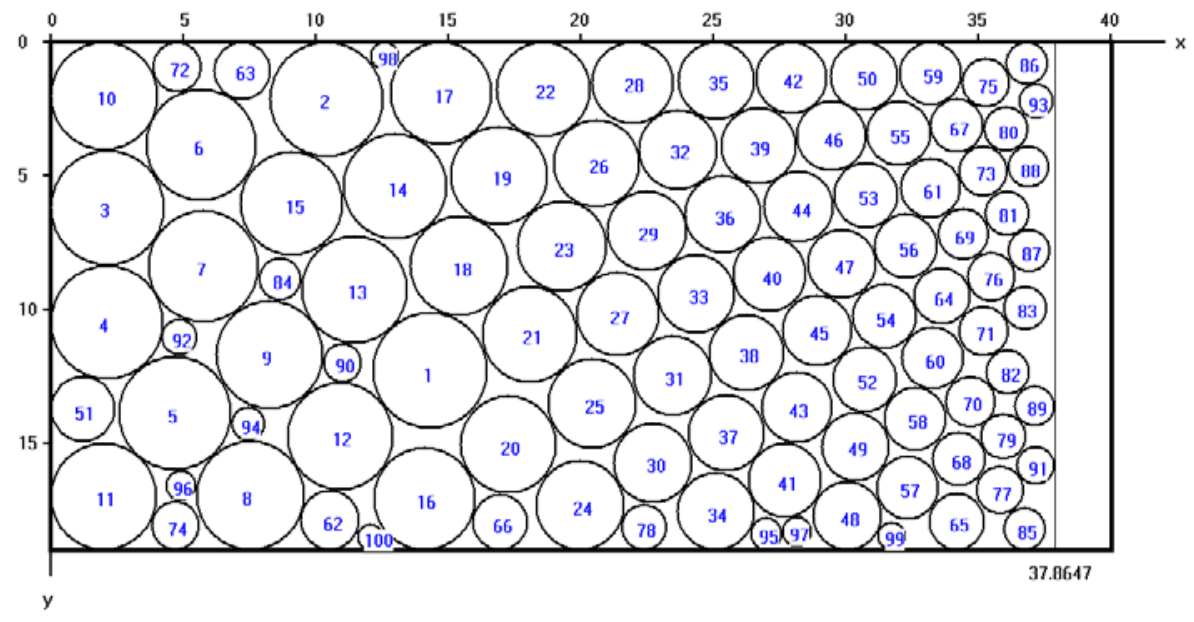

Fig. 8 A packing layout of SY6 from ExOrd2 $(L=37.8647)$

From Tables 3 to 5, it can be observed that overall ExOrd1 outperforms the decreasing order of radii and ExOrd2 further improves the performance of ExOrd1. For a certain instance, by applying the heuristic of decreasing order of the circles' radii and then applying ExOrd1 followed by ExOrd2, with a complexity of $O(2 n)$, the solution quality can be improved quickly. It is hence worthwhile applying these heuristics to the initial generation of a genetic algorithm.

Actually, inspired by ExOrd1 and ExOrd2 where minor-adjustment to a circle sequence may improve the packing quality, we anticipate that similar minor-adjustment to the current best solution(s) at each iteration in a genetic algorithm may also produce better solutions. The minor-adjustment (insert-mutation) can be executed to the sole best solution, or to a small part of the better solutions within each generation of the genetic algorithm.

It can also be noted from Tables 3 to 5 that among the three approaches, the first criterion $(x)$ behaves the best, and $\left(d_{\min }+x\right)$ the second best. Performances of the other three criteria are not so good. Our numerical experiments in Section 5 draw the similar conclusion for these three criteria. 


\section{Improved Genetic Algorithm}

In a genetic algorithm (GA), solutions to the problem are represented by chromosomes of genetic structure (genotype). Each chromosome is evaluated by a function or through a procedure to change the chromosome into a visual or delomorphic solution (phenotype) with an objective value (e.g. change it into a packing configuration with a length of the rectangle in this work). At the beginning of the GA, an initial generation of chromosomes is randomly produced. Throughout the genetic evolution, by the mechanism of selection, crossover and mutation, good-quality offspring are born from the previous generation (parents). Generation by generation, the stronger chromosomes are the survivors in a competitive environment. At the end of the GA, optimal or near-optimal solutions can be achieved.

Selection, crossover and mutation are the crucial operators of the GA. The selection operator is an artificial mechanism based on natural selection (i.e. the weakest individuals die off, the fittest proliferate), which makes future generations hopefully become fitter and fitter. The crossover operator makes chromosomes mate to produce new offspring. Crossover constitutes the information exchange phase of the GA in which diversity and innovation are produced within the chromosomes. The mutation operator generates random change to a number of the chromosomes within each generation.

\subsection{Standard GA Procedure}

A standard procedure of GA can be usually depicted as Fig. 9. At each iteration, a new generation will be produced by crossover, mutation and selection, and the population size, popsize, is usually kept constant.

Produce an initial generation comprising popsize chromosomes, and evaluate all the chromosomes using the decoding procedure, $t=1$;

do $\{1$. Selection: Select the xsize best chromosomes (according to their objective value) in each generation to crossover, and the msize worst chromosomes to mutate;

2. Crossover and Mutation: Conduct crossover to the xsize best chromosomes generating xsize new chromosomes; conduct mutation to the msize worst chromosomes generating msize new chromosomes;

3. The offspring (xsize+msize new chromosomes) from crossover and mutation are decoded. Sort the parents and the offspring according to their objective values, and select the former popsize best chromosomes as the new generation;

4. $t++$.

\} until satisfy the termination condition;

Output the best solution.

Fig. 9 A standard procedure of GA 
Selection: Common selection methods include roulette-wheel selection [7] and tournament method [8]. Although roulette-wheel selection is one commonly used technique, tournament method is adopted in our GA. The reason is that in the tournament method the objective value of a chromosome can be directly used as the selection criterion, but in the roulette-wheel selection the fitness of a chromosome is always used as the selection criterion.

Assume that the number of the chromosomes that are selected to crossover is xsize; the number of the chromosomes that are selected to mutate is msize. We can let $x$ size + msize $=$ popsize. The ratio, $C_{r}=x$ xize/popsize, is called crossover rate, often $C_{r} \in[0.5,0.9]$; and the ratio, $M_{r}=$ msize/popsize, is called mutation rate, often $M_{r} \in[0.1,0.3]$. Usually, $C_{r}+M_{r}=1.0 . C_{r}$ and $M_{r}$ are two important parameters that influence the convergence of GA. In general, when $M_{r}$ increases, GA will converge to the final solution slowly, so that GA has more chances to explore the search space to find better solutions.

Crossover: Poon and Carter [15] presented a survey of crossover operators for ordering applications. Regarding the permutation-based representation, the following crossover operators have been widely used: partially matched crossover (PMX), intended to keep the absolute positions of elements, and linear order crossover (LOX), intended to respect relative positions. PMX is adopted in our GA.

Mutation: There are a number of methods to mutate [5], such as insertion, swap and reversion. Insertion and swap is employed in our GA.

Termination Condition: The termination condition for the GA in our work is that the algorithm stops when the objective value difference between the worst chromosome and the best one in the current generation is equal to or less than 0.0001 .

\subsection{Improved GA for Circle Packing}

We find that packing non-identical circles into a rectangle with open length is much more challenging than the common combinatorial problems like typical production scheduling problems and the bin packing problem. The toughness exhibits in several aspects: (1) Bigger difference exists between various position selection criteria; (2) The deviation of solutions from different tests for the same instance is prominent; (3) The solution time for the moderate-size onward cases is very long. Therefore, there is still a big room for further improvement on the existing solution techniques.

In addition to using the suitable position selection criterion in the packing procedure, we further improved the GA itself. Fig. 10 shows the improved GA (IGA), the following adaptations and special settings have been conducted.

Initial Generation Followed by Exchange-Order Operations. A quick good solution before evolution can save some valuable search time. With this purpose, the sequence in decreasing order of the circles' radii is put into the initial generation followed by the exchange-order operations. 
Produce an initial generation comprising (popsize-1) random chromosomes, and evaluate all the chromosomes using the decoding procedure (i.e. the packing procedure described in §3.2);

A chromosome from the decreasing radii order is put into the initial generation followed by the exchange-order operations;

$t=1$

do $\{1$. Selection: Select the xsize best chromosomes in each generation to crossover, and the msize worst chromosomes to mutate $(x s i z e+$ msize $=$ popsize $)$;

2. Insert-mutation to top $10 \%$ best chromosomes;

3. Crossover with lower $\boldsymbol{C}_{\boldsymbol{r}}=$ xsize/popsize and swap mutation with higher $\boldsymbol{M}_{\boldsymbol{r}}=$ msize/popsize;

4. The offspring from crossover and mutations are decoded. Sort the parents and the offspring according to their objective values, and select the former popsize best chromosomes as the new generation;

5. $t++$.

\} until satisfy the termination condition;

Output the best solution.

Fig. 10 The improved GA with adaptations and special settings

Insert-Mutation for Better Chromosomes. In the standard GA procedure as shown in Fig. 9, the operation of selection is followed by the crossover and the mutation to produce the next generation. In our improved GA, a local search operation - an insert-mutation is added between the selection and the crossover. This operation is applied to the top $10 \%$ best individuals. As well-known, the insert-mutation has the minimum destructiveness to a chromosome compared with swap and reversion, while reversion has the maximum destructiveness. The insert-mutation, similar to the exchange-order operations, may produce better solutions from the top $10 \%$ best solutions. Such a minor-adjustment operator benefits the algorithm in terms of solution speed and quality.

Low Crossover Rate and High Mutation Rate. In the common use of GA for other problems, like scheduling and bin packing, a higher crossover rate, $C_{r} \in[0.5,0.9]$, and a lower mutation rate, $M_{r} \in[0.1,0.3]$ are set. However, for the challenging non-identical circles packing problem, a low crossover rate but a very high mutation rate benefit the solution process (see Section 5). The reason for this is that, the high mutation rate increases the diversity in a new generation, and thus maintains the evolution process for a long time and makes it more possible to achieve much better solutions. In fact, the high mutation rate is also adopted in the work on three-dimensional bin packing [18].

Large Population of Each Generation. In order to get better solutions, a direct way is to increase the number of individuals (popsize) in each generation. However, the effectiveness of the large population should be evaluated, considering the cost of longer search time. From our experience, the population size should not be too large, because exceeding a threshold number, the growing of population size exerts little impact on the improvement of the final solution. In fact, there is a trade-off between the high mutation rate and the large population, which can be observed in the following computational experiments. 


\section{Computational Experiments}

In this section, computational experiments are conducted to test the performance of the proposed algorithm and the heuristics combined. The proposed genetic algorithm, together with the corresponding heuristics and decoding procedure, is coded in C language and compiled with Microsoft Visual VC++ 8.0. All the numerical experiments are conducted on a computer with an Intel T7500 2.20GHz Duo CPU and 1.96GB RAM. The search time is measured in seconds.

A sample solution by IGA is first presented, followed by the six instances SY1 to SY6 used in Stoyan and Yaskov [16, 17]. Results are compared with those from existing methods in the literature. This is to check the performance of different position selection criteria and fine-tune the parameters of IGA. Moderate- and large-size instances (SY12 to SY1234) are solved subsequently.

\subsection{A Sample Solution by IGA}

Table 6. A sample solution obtained by IGA with the first selection criterion

\begin{tabular}{|c|c|c|c|c|c|c|c|c|c|}
\hline$i$ & $\pi_{i}$ & $r_{i}$ & $x_{i}$ & $y_{i}$ & $s_{1}$ & $S_{2}$ & $s_{3}$ & $a d j_{1}$ & $a d j_{2}$ \\
\hline 1 & 6 & 4.0 & 4.0000 & 4.0000 & 1 & 1 & 0 & 0 & 0 \\
\hline 2 & 1 & 6.0 & 6.0000 & 24.0000 & 0 & 1 & 1 & 0 & 0 \\
\hline 3 & 3 & 5.0 & 5.0000 & 12.9443 & 0 & 1 & 0 & 6 & 0 \\
\hline 4 & 2 & 5.5 & 13.1966 & 6.3818 & 0 & 0 & 0 & 6 & 3 \\
\hline 5 & 4 & 4.8 & 14.0226 & 16.7696 & 0 & 0 & 0 & 3 & 1 \\
\hline 6 & 5 & 4.3 & 16.1587 & 25.7000 & 0 & 0 & 1 & 1 & 0 \\
\hline 7 & 7 & 3.8 & 20.8950 & 11.5995 & 0 & 0 & 0 & 2 & 4 \\
\hline 8 & 9 & 2.9 & 20.8410 & 2.9000 & 1 & 0 & 0 & 2 & 0 \\
\hline 9 & 8 & 3.3 & 21.3736 & 20.1714 & 0 & 0 & 0 & 5 & 4 \\
\hline 10 & 10 & 2.3 & 22.4484 & 27.7000 & 0 & 0 & 1 & 5 & 0 \\
\hline \multicolumn{10}{|c|}{$N=10, W=30, L=24.7484$, area utilization $U=79.4694 \%$, search time $T=1.578(\mathrm{~s})$} \\
\hline
\end{tabular}

Table 6 presents a sample solution obtained by IGA with the first position selection criterion $(x)$, where the parameter settings are popsize $=200, C_{r}: M_{r}=3: 7$. One may notice that, in the solution obtained by IGA, besides the coordinates $\left(x_{i}, y_{i}\right)$ of the circle center, the relative position $\left(s_{1}, s_{2}, s_{3}\right.$, $\left.a d j_{1}, a d j_{2}\right)$ that tells which two items each circle is tangent to is given. The relative positions have practical significance in the case of manual packing. This feature is inherited from our previous work on three-dimensional bin packing by He et al. [9].

\subsection{Tests with SY1 to SY6}

To test the behavior of different position selection criteria in solving the instances via IGA, the algorithm combined with each criterion is executed to solve the six instances SY1 to SY6 used in Stoyan and Yaskov $[16,17]$. Table 7 reports the results, where popsize $=200, C_{r}: M_{r}=2: 8$ for all the tests. For each of the instances SY1 to SY4, 20 runs of the algorithm are executed under each 
selection criterion, and the best solution is reported here; for SY5 and SY6, 5 runs are conducted with each criterion, and the best solution is reported here. Under each criterion, $T$ refers to the corresponding search time in seconds for the run which obtained the best solution for an instance. As stated earlier, with the parameter setting of lower crossover rate and higher mutation rate, the proposed GA is observed to demonstrate good performance. This is the reason why we set popsize $=200, C_{r}: M_{r}=2: 8$ for all the tests.

From Table 7, we can observe that, the first criterion $(x)$ still behaves the best, $\left(d_{\min }+x\right)$ the second best, and the other three criteria are not so good. This is consistent with their performance in packing the circle sequence of decreasing of the circles’ radii, as well as in ExOrd1 and ExOrd2. So far, we could conclude that the first criterion of the minimum $x$ coordinate is the best one among all the criteria considered. Hence, we subsequently test the proposed algorithm combined with the first criterion under various parameter settings, popsize and $C_{r}: M_{r}$, (see Table 8), and compare our best results with those provided in the literature (refer to Table 9).

Table 7. Results of SY1 to SY6 by IGA combined with diverse selection criteria $\left(\right.$ popsize $\left.=200, C_{r}: M_{r}=2: 8\right)$

\begin{tabular}{|c|r|r|r|r|r|r|r|r|r|r|r|r|}
\hline \multicolumn{4}{|c|}{ Criteria } & \multicolumn{3}{|c|}{$x$} & \multicolumn{3}{c|}{$\lambda$} & \multicolumn{2}{c|}{$x$ and $\lambda$} & \multicolumn{3}{|c|}{$\lambda / x$} & $d_{\min }+x$ \\
\hline \#Inst & $N$ & $W$ & $L_{x}$ & \multicolumn{1}{c|}{$T_{x}$} & $L_{\lambda}$ & $T_{\lambda}$ & $L_{(x \text { and } \lambda)}$ & $T_{(x \text { and } \lambda)}$ & $L_{\lambda / x}$ & \multicolumn{1}{c|}{$T_{\lambda / x}$} & & \\
\hline SY1 & 30 & 9.5 & 17.3185 & 389.38 & 17.4023 & 430.36 & 17.4883 & 555.41 & 17.4814 & 548.78 & 17.4634 & 253.73 \\
\hline SY2 & 20 & 8.5 & 14.6597 & 56.33 & 14.7009 & 69.86 & $\mathbf{1 4 . 6 1 6 3}$ & 83.17 & 14.7137 & 75.92 & 14.6345 & 23.375 \\
\hline SY3 & 25 & 9.0 & $\mathbf{1 4 . 5 1 2 9}$ & 204.83 & 14.6833 & 137.11 & $\mathbf{1 4 . 6 2 2 2}$ & 166.29 & 14.6957 & 147.20 & $\mathbf{1 4 . 5 3 1 8}$ & 135.92 \\
\hline SY4 & 35 & 11.0 & $\mathbf{2 3 . 6 6 0 9}$ & 605.48 & 23.7358 & 638.72 & $\mathbf{2 3 . 7 5 5 5}$ & 606.29 & 23.8616 & 718.81 & 23.7688 & 409.80 \\
\hline SY5 & 100 & 15.0 & 36.7300 & $17,756.00$ & 37.3882 & 9338.00 & 37.1829 & 24241.50 & 37.3116 & 17948.77 & 37.0789 & 15605.14 \\
\hline SY6 & 100 & 19.0 & 37.0411 & $17,103.41$ & 38.2728 & 8767.50 & 38.0797 & 19393.23 & 38.1802 & 9230.36 & 37.3435 & 23199.95 \\
\hline Mean & & & $\mathbf{2 3 . 9 8 7 2}$ & $6,019.24$ & 24.3639 & 3230.26 & 24.2908 & 7507.65 & 24.3740 & 4778.31 & 24.1368 & 6604.653 \\
\hline
\end{tabular}

Table 8 presents the results of SY1 to SY6 by IGA combined with the first position selection criterion. For each of the instances SY1 to SY4, the best solution out of 20 runs under each parameter setting is presented; for SY5 and SY6, the best solution out of 5 runs with each setting is presented. We first keep popsize $=200$, and change the ratio of $C_{r}: M_{r}$ from 8:2 to 2:8. It can be seen that with the decreasing of $C_{r}: M_{r}$, the search time increases and the solution quality becomes better and better, obviously indicated by the mean objective values of $L$. Then we compare the performances between higher mutation rate $\left(C_{r}: M_{r}=1: 9\right.$, popsize $\left.=400\right)$ and large population sizes $\left(C_{r}: M_{r}=3: 7\right.$, popsize $=1000$ and 2000 respectively $)$. As the population size grows from popsize $=$ 200 to 1000 and then to 2000 (but keep $C_{r}: M_{r}=3: 7$ ), the solution quality improves but at the cost of over 20 -fold average search time $(75192.31 / 3145.52=23.91)$. In contrast, the case with $C_{r}: M_{r}=1: 9$ and popsize $=400$ witnesses very good solution quality and relatively short search time $(75192.31 / 29432.27=2.55)$. Therefore, it is fair to say that the higher mutation rate brings about more benefits than the large number of population does.

Table 9 presents the comparative results of the existing methods and the proposed algorithm, 
where the subscript SY means the results given by Stoyan and Yaskov [17], HM means those by Hifi and M'Hallah [10], B1.0 and B1.5 are the results by the heuristic methods developed by Huang et al. [12] (but the results were given by Akeb and Hifi [1]), $A H$ means the best solutions obtained by Akeb and Hifi [1]. In the last six columns of Table 9, the best solutions by the proposed method (IGA) are reported. Figs. A1 to A6 in Appendix display the packing configurations of the best solutions of SY1 to SY6 achieved by IGA.

It is rather difficult to compare the performance of different methods due to the differences in the programming languages used, computer hardware deployed, and sometimes lack of data reported. Therefore, we report the hardware used by researchers. Stoyan and Yaskov [17] did not present the search time of their algorithm for SY1 to SY6, except that about 1h was taken to obtain the solution of SY6 on an IBM PC/AT 486. The search time of the GA by Hifi and M'Hallah [10] was measured on a Pentium III 733MHz and 128M of RAM. Akeb and Hifi [1] reported the results from B1.0, B1.5 and their beam search from computational tests executed on an Intel Celeron, $3 \mathrm{GHz}$ and $256 \mathrm{Mb}$ of RAM.

Furthermore, the algorithmic feature of all methods should be also recapitulated. Stoyan and Yaskov [17] used a mathematical model combining a tree-search procedure and a reduced gradient method, belonging to exact domain. The genetic algorithm integrated with a constructive procedure proposed by Hifi and M'Hallah [10] is a stochastic search method. But the heuristic methods developed by Huang et al. [12] are deterministic and yet enumerative methods with high computational complexity up to $O\left(n^{10}\right)$, this is the reason why their techniques are so timeconsuming (the dash “-” in Table 9 means over 30 hours). The adaptive beam search presented by Akeb and Hifi [1] based on their previous work is still a stochastic search method involving heuristics. The proposed method (IGA) in the present work is obviously stochastic with heuristics.

In order to evaluate the performance of the methods in solving the instances of different sizes, we classify the instances investigated into two groups: put SY1 to SY4 into the small-size group (20 $\leq N \leq 40)$, and SY5 and SY6 into the large-size $(N \geq 70)$. And let the moderate-size group have a number of circles $(40<N<70$ ). For the reasons mentioned earlier, the comparison and analysis of Table 9 focus on the ultimate packing results achieved with a side look at the computational time used by different approaches. The following aspects are observed:

(1) B1.0 has a faster solution speed but with lower solution quality than AH beam search for the small-size group; B1.5 obtains a higher average solution quality but with much longer search time than AH beam search for the small-size group.

(2) IGA achieves better solutions within shorter search time than AH beam search for all small-size instances (SY1 to SY4), and obtains two better solutions (for SY1 and SY4) than B1.5.

(3) For the large-size instances, AH beam search shows more superiority in terms of both solution speed and solution quality. 
So far, the aim of this study to challenge the existing methods in the literature is partially realized in solving the small-size instances, but for the moderate- and large-size instances, we need to dedicate more innovative efforts, such as novel decomposition techniques and other efficient meta-heuristics. We could boldly predict that if the idea of three-type positions and the best criterion for position selection were integrated with the beam search, the search ability might improve further. As well known, the genetic algorithm has the defect that its search ability degrades quickly with growing of the problem size. 
Table 8. Results of SY1 to SY6 by IGA combined with the first position selection criterion (the minimum $x$ coordinate)

\begin{tabular}{|c|c|c|c|c|c|c|c|c|c|c|c|c|c|c|c|c|c|c|}
\hline \multicolumn{3}{|c|}{$\left(\right.$ popsize $\left., C_{r}: M_{r}\right)$} & \multicolumn{2}{|c|}{$(200,8: 2)$} & \multicolumn{2}{|c|}{$(200,5: 5)$} & \multicolumn{2}{|c|}{$(200,4: 6)$} & \multicolumn{2}{|c|}{$(200,3: 7)$} & \multicolumn{2}{|c|}{$(200,2: 8)$} & \multicolumn{2}{|c|}{$(400,1: 9)$} & \multicolumn{2}{|c|}{$(1000,3: 7)$} & \multicolumn{2}{|c|}{$(2000,3: 7)$} \\
\hline \#Inst & $N$ & $W$ & $L_{1}$ & $T_{1}$ & $L_{2}$ & $T_{2}$ & $L_{3}$ & $T_{3}$ & $L_{4}$ & $T_{4}$ & $L_{5}$ & $T_{5}$ & $L_{6}$ & $T_{6}$ & $L_{7}$ & $T_{7}$ & $L_{8}$ & $T_{8}$ \\
\hline SY1 & 30 & 9.5 & 17.5412 & 66.31 & 17.5060 & 96.91 & 17.4209 & 135.56 & 17.3389 & 206.70 & 17.3185 & 389.38 & 17.2297 & 937.40 & 17.3166 & 1422.25 & 17.2255 & 4182.19 \\
\hline SY2 & 20 & 8.5 & 14.7703 & 14.11 & 14.6903 & 22.05 & 14.6860 & 20.03 & 14.6777 & 35.86 & 14.6597 & 56.33 & 14.5597 & 112.09 & 14.5758 & 516.06 & 14.5406 & 676.74 \\
\hline SY3 & 25 & 9.0 & 14.6514 & 37.64 & 14.5911 & 80.53 & 14.5630 & 93.48 & 14.5561 & 102.47 & 14.5129 & 204.83 & 14.4994 & 182.95 & 14.4861 & 453.55 & 14.4768 & 1962.20 \\
\hline SY4 & 35 & 11.0 & 24.1177 & 131.52 & 24.0355 & 140.86 & 23.9653 & 293.48 & 23.7569 & 336.69 & 23.6609 & 605.48 & 23.5477 & 1380.22 & 23.6572 & 2762.04 & 23.5878 & 8166.17 \\
\hline SY5 & 100 & 15.0 & 37.2062 & 1223.03 & 37.0343 & 5064.188 & 36.8934 & 6090.70 & 36.8217 & 9674.68 & 36.7300 & 17756.00 & 36.4508 & 88653.44 & 36.6879 & 143454.34 & 36.6053 & 195815.40 \\
\hline SY6 & 100 & 19.0 & 37.6659 & 2041.08 & 37.4211 & 6527.14 & 37.3426 & 7649.78 & 37.2759 & 8516.72 & 37.0411 & 17103.41 & 36.9693 & 85327.50 & 36.9570 & 111841.19 & 36.9228 & 240351.17 \\
\hline Mean & & & 24.3255 & & 24.2131 & & 24.1452 & & 24.0712 & 3145.52 & 23.9872 & & 23.8761 & 29432.27 & 23.9468 & & 23.8931 & 75192.31 \\
\hline
\end{tabular}

Table 9. Results of SY1 to SY6 by the existing methods and the proposed IGA with the first position selection criterion

\begin{tabular}{|c|c|c|c|c|c|c|c|c|c|c|c|c|c|c|c|c|c|c|}
\hline \multirow[b]{2}{*}{ \#Inst } & \multirow[b]{2}{*}{$N$} & \multirow[b]{2}{*}{$W$} & \multicolumn{10}{|c|}{ Results from the literature } & \multicolumn{6}{|c|}{ Results by IGA } \\
\hline & & & $L_{S Y}$ & $T_{S Y}$ & $L_{H M}$ & $T_{H M}$ & $L_{B 1.0}$ & $T_{B 1.0}$ & $L_{B 1.5}$ & $T_{B 1.5}$ & $L_{A H}$ & $T_{A H}$ & $L_{6}$ & $T_{6}$ & $L_{7}$ & $T_{7}$ & $L_{8}$ & $T_{8}$ \\
\hline SY1 & 30 & 9.5 & 17.491 & & 17.9719 & 25 & 17.561 & 291.90 & 17.291 & - & 17.2315 & 1010.02 & $\mathbf{1 7 . 2 2 9 7}$ & 937.40 & 17.3166 & 1422.25 & 17.2255* & $4182.1 \mathrm{C}$ \\
\hline SY2 & 20 & 8.5 & 14.895 & & 15.2294 & 9 & 14.735 & 27.46 & 14.535 & 22396 & 14.6277 & 320.29 & 14.5597 & 112.09 & 14.5758 & 516.06 & 14.5406* & 676.7 \\
\hline SY3 & 25 & 9.0 & 14.930 & & 14.9376 & 20 & 14.660 & 107.67 & 14.470 & - & 14.5310 & 544.56 & 14.4994 & 182.95 & 14.4861 & 453.55 & 14.4768* & 1962.20 \\
\hline SY4 & 35 & 11.0 & 24.355 & & 24.7145 & 15 & 23.915 & 875.10 & 23.555 & - & 23.6719 & 1482.09 & 23.5477* & 1380.22 & 23.6572 & 2762.04 & 23.5878 & 8166.17 \\
\hline SY5 & 100 & 15.0 & 38.047 & & 38.0470 & ? & 36.547 & - & 36.327 & - & 36.0796 & 27606.91 & $36.4508^{*}$ & 88653.44 & 36.6879 & 143454.34 & 36.6053 & 195815.40 \\
\hline SY6 & 100 & 19.0 & 38.647 & $\approx 1 \mathrm{~h}$ & 38.6470 & 287 & 36.997 & - & 36.857 & - & 36.8456 & 28298.84 & 36.9693 & 85327.50 & 36.9570 & 111841.19 & 36.9228* & 240351.17 \\
\hline Mean & & & 24.728 & & 24.9246 & & 24.069 & & 23.839 & & 23.8312 & & 23.8762 & & 23.9468 & & 23.8931 & \\
\hline
\end{tabular}

*the best solutions found by IGA (see Figs. A1 to A6) 
Table 10. Results of all SY instances solved directly by IGA with the first criterion (the minimum $x$ coordinate)

\begin{tabular}{|c|c|c|c|c|c|c|c|c|c|c|c|c|c|c|}
\hline \multicolumn{5}{|c|}{$\left(\right.$ popsize, $\left.C_{r}: M_{r}\right)$} & \multicolumn{2}{|c|}{$(200,3: 7)$} & \multicolumn{2}{|c|}{$(200,2: 8)$} & \multicolumn{2}{|c|}{$(400,1: 9)$} & \multicolumn{2}{|c|}{$(1000,3: 7)$} & \multicolumn{2}{|c|}{$(2000,3: 7)$} \\
\hline & $N$ & $W$ & $L_{A H}$ & $T_{A H}$ & $L_{4}$ & $T_{4}$ & $L_{5}$ & $T_{5}$ & $L_{6}$ & $T_{6}$ & $L_{7}$ & $T_{7}$ & $L_{8}$ & $T_{8}$ \\
\hline SY1 & 30 & 9.5 & 17.2315 & 1010.02 & 17.3389 & 206.70 & 17.3185 & 389.38 & 17.2297 & 937.40 & 17.3166 & $1,422.25$ & 17.2255 & $4,182.19$ \\
\hline SY2 & 20 & 8.5 & 14.6277 & 320.29 & 14.6777 & 35.86 & 14.6597 & 56.33 & 14.5597 & 112.09 & 14.5758 & 516.06 & 14.5406 & 676.74 \\
\hline SY3 & 25 & 9.0 & 14.5310 & 544.56 & 14.5561 & 102.47 & 14.5129 & 204.83 & 14.4994 & 182.95 & 14.4861 & 453.55 & 14.4768 & $1,962.20$ \\
\hline SY4 & 35 & 11.0 & 23.6719 & 1482.09 & 23.7569 & 336.69 & 23.6609 & 605.48 & 23.5477 & 1380.22 & 23.6572 & $2,762.04$ & 23.5878 & $8,166.17$ \\
\hline SY5 & 100 & 15.0 & 36.0796 & $27,606.91$ & 36.8217 & $9,674.68$ & 36.7300 & $17,756.00$ & 36.4508 & 88653.44 & 36.6879 & $143,454.34$ & 36.6053 & $195,815.40$ \\
\hline SY6 & 100 & 19.0 & 36.8456 & $28,298.84$ & 37.2759 & $8,516.72$ & 37.0411 & $17,103.41$ & 36.9693 & 85327.50 & 36.9570 & $111,841.19$ & 36.9228 & $240,351.17$ \\
\hline SY12 & 50 & 9.5 & 29.7011 & 3778.68 & 30.3629 & 1886.02 & 30.1558 & 1662.49 & 30.1557 & 10152.43 & 30.2187 & 12523.08 & NA & NA \\
\hline SY13 & 55 & 9.5 & 30.6371 & 4733.77 & 31.1333 & 957.438 & 31.0730 & 2455.78 & 30.9792 & 11886.67 & 30.8302 & 19106.22 & NA & NA \\
\hline SY14 & 65 & 11.0 & 38.0922 & 7657.32 & 38.5323 & 5346.59 & 38.4746 & 5388.53 & 38.3302 & 22521.23 & 38.3618 & 29225.59 & NA & NA \\
\hline SY23 & 45 & 9.0 & 27.8708 & 2575.86 & 28.1944 & 1338.00 & 28.2253 & 734.89 & 28.0146 & 5617.73 & 28.1365 & 4203.98 & NA & NA \\
\hline SY24 & 55 & 11.0 & 34.5476 & 4913.31 & 34.9733 & 1008.66 & 34.8459 & 2551.97 & 34.8276 & 13018.11 & 34.8814 & 10731.41 & NA & NA \\
\hline SY34 & 60 & 11.0 & 34.9354 & 5698.15 & 35.4986 & 2279.41 & 35.4469 & 4420.22 & 35.3146 & 16333.64 & 35.2236 & 13402.69 & NA & NA \\
\hline SY56 & 200 & 19.0 & 64.7246 & 181457.31 & 66.3029 & 46755.28 & 66.2043 & 120757.82 & 65.6338 & 303555.91 & 65.4783 & 401414.67 & NA & NA \\
\hline SY123 & 75 & 9.5 & 43.2558 & 10479.80 & 44.1389 & \begin{tabular}{|l}
3796.45 \\
\end{tabular} & 44.0582 & 8010.11 & 44.1445 & 19894.11 & 44.0697 & 31593.08 & NA & NA \\
\hline SY124 & 85 & 11.0 & 48.8927 & 15237.25 & 50.0167 & 10144.23 & 49.9637 & 11222.00 & 49.7550 & 65641.93 & 49.7761 & 51422.38 & NA & NA \\
\hline SY134 & 90 & 11.0 & 49.3954 & 17548.61 & 50.6144 & 7763.25 & 50.3137 & 17710.77 & 50.5128 & 63387.25 & 50.5544 & 22083.88 & NA & NA \\
\hline SY234 & 80 & 11.0 & 45.9526 & 12791.49 & 46.7388 & 5168.02 & 46.4927 & 7578.187 & 46.4929 & 40461.20 & 46.4994 & 36652.73 & NA & NA \\
\hline SY1234 & 110 & 11.0 & 60.2613 & 29952.11 & 61.8524 & 19827.45 & 61.5723 & 51684.78 & 61.1962 & 116776.28 & 61.3166 & 97861.94 & NA & NA \\
\hline mean & & & 42.3556 & 24735.31 & 43.1966 & 8855.9 & 43.0689 & 19514.796 & 42.9464 & 57437.21 & 42.9456 & 60851.8 & & \\
\hline
\end{tabular}




\subsection{Tests with the Moderate and Large Instances SY12 to SY1234}

One may observe that the results of other twelve moderate- and large-size instances have not been presented yet. Indeed, we have directly solved these instances with IGA (see Table 10, for each instance of SY12 to SY1234, three runs of the algorithm have been executed under each parameter setting, and the best solution is presented here). Considering the instance size and GA's degradation, we do not think that the direct application of the algorithm to these instances is a good choice. Instead, decomposition is more effective and efficient in solving the instances of such scale, as seen in $\S 5.4$.

\subsection{Decomposition of the Moderate and Large Instances}

Considering the long search time to solve the moderate- and large-size instances, we can use a simple decomposition method to obtain an aggregate packing solution for each instance, instead of directly solving it with the proposed algorithm. For example, to get a packing solution of the instance SY12 ( $W=9.5$ ), we can merge the solution of SY1 and the solution of SY2 with new width $W=9.5$, denoted by SY2(9.5), which can be easily solved by the proposed algorithm. So the length of SY12 is $17.3389+13.0917=30.4306$ in the case $\left(\right.$ popsize, $\left.C_{r}: M_{r}\right)=(200,3: 7)$. Other instances can be handled similarly.

Table 11 presents the results of SY1 to SY3 and SY5 with new widths (for each instance with the new width, 20 runs of IGA have been executed under each parameter setting, and the best solution is presented here). With the results of SY1 to SY6 (see the upper part of Table 10) and the solutions available in Tables 11, we can obtain the aggregate solutions for SY12 to SY1234, as shown in Table 12. From Table 12, it can be seen that the average solution time is much shorter, but the solution quality sacrifices very little. For example, $L_{6 d}$ has an average value of $\mathbf{4 3 . 0 5 2 5}$ in Table 12, only a little bit higher than the average value of $L_{6}, \mathbf{4 2 . 9 4 6 4}$ in Table 10.

Table 11. Results SY1 to SY3 and SY5 with new widths solved by IGA under diverse parameter settings

\begin{tabular}{|c|c|c|c|c|c|c|c|c|c|c|c|c|}
\hline \multicolumn{3}{|c|}{$\left(\right.$ popsize $\left., C_{r}: M_{r}\right)$} & \multicolumn{2}{|c|}{$(200,3: 7)$} & \multicolumn{2}{|c|}{$(200,2: 8)$} & \multicolumn{2}{|c|}{$(400,1: 9)$} & \multicolumn{2}{|c|}{$(1000,3: 7)$} & \multicolumn{2}{|c|}{$(2000,3: 7)$} \\
\hline & $N$ & $W$ & $L_{4}$ & $T_{4}$ & $L_{5}$ & $T_{5}$ & $L_{6}$ & $T_{6}$ & $L_{7}$ & $T_{7}$ & $L_{8}$ & $T_{8}$ \\
\hline SY2(9.5) & 20 & 9.5 & 13.0917 & 51.16 & 13.0428 & & 13.0450 & 89.03 & 13.0428 & 304.75 & 12.9831 & 786.53 \\
\hline SY3(9.5) & 25 & 9.5 & 13.8539 & & 13.8527 & 78.44 & & 449.09 & 13.8390 & 332.30 & 13.7799 & 739.84 \\
\hline SY1(11.0) & 30 & 11.0 & 15.0488 & 224.20 & 14.9784 & 380.69 & 14.9527 & 865.61 & 14.9533 & 1260.56 & 14.8611 & 2115.84 \\
\hline SY2(9.0) & 20 & 9.0 & 13.9386 & 30.59 & 13.9012 & 81.75 & 13.6708 & 246.27 & 13.7446 & 486.84 & 13.7038 & 105030 \\
\hline SY2(11.0) & 20 & & & & & & & & & & & 1202.13 \\
\hline SY3(11.0) & 25 & 11.0 & 11.9324 & 98.70 & 11.9231 & & 11.9113 & 259.22 & 11.8995 & 524.08 & 11.8740 & 732.52 \\
\hline SY5(19.0) & 100 & 19.0 & 29.0124 & 10640.58 & 28.9862 & 25288.75 & 28.8962 & 67280.78 & 28.9323 & 105303.75 & 28.8115 & 206538.80 \\
\hline
\end{tabular}

In fact, in Table 12 the search time for SY56 occupies a great proportion (>80\%) of the total time. If SY56 $(N=200)$ is broken into 4 or 5 parts for the aggregate solution, the average search time can decline tremendously to $3 \mathrm{~h}$. In Table 12, the mean search times after singling out SY56 
(denoted by mean*) are much shorter than the before. Under the parameter setting, (popsize, $C_{r}: M_{r}$ ) $=(400,1: 9)$, an instance with $N \leq 50$ (like SY23) can be solved within 2000s; Hence, we can estimate that the aggregate solution of SY56 can be obtained within 10000s (2.78h), while other instances are solved within an hour. In short, the search time can reduce by one order of magnitude via decomposition, but the solution quality sacrifices very little. It should be noted that the decomposition takes the advantage of the proposed method in solving the small-size instances.

Table 12. Aggregate solutions of SY12 to SY1234 obtained by the simple decomposition method

\begin{tabular}{|c|c|c|c|c|c|c|c|c|c|c|c|c|}
\hline \multicolumn{3}{|c|}{$\left(\right.$ popsize, $C_{r}: M_{r}$ ) } & \multicolumn{2}{|c|}{$(200,3: 7)$} & \multicolumn{2}{|c|}{$(200,2: 8)$} & \multicolumn{2}{|c|}{$(400,1: 9)$} & \multicolumn{2}{|c|}{$(1000,3: 7)$} & \multicolumn{2}{|c|}{$(2000,3: 7)$} \\
\hline & $N$ & $W$ & $L_{4 d}$ & $T_{4 d}$ & $L_{5 d}$ & $T_{5 d}$ & $L_{6 d}$ & $T_{6 d}$ & $L_{7 d}$ & $T_{7 d}$ & $L_{8 d}$ & $T_{8 d}$ \\
\hline SY12 & 50 & 9.5 & 30.4306 & 257.86 & 30.3613 & 454.58 & 30.2747 & 1026.43 & 30.3594 & 1727.00 & 30.2086 & 4968.72 \\
\hline SY13 & 55 & 9.5 & 31.1928 & 273.68 & 31.1712 & 467.82 & 30.9930 & 1386.49 & 31.1556 & 1754.55 & 31.0054 & 4922.03 \\
\hline SY14 & 65 & 11.0 & 38.8057 & 560.89 & 38.6393 & 986.17 & 38.5004 & 2245.83 & 38.6105 & 4022.60 & 38.4489 & 10282.01 \\
\hline SY23 & 45 & 9.0 & 28.4947 & 133.06 & 28.4141 & 286.58 & 28.1702 & 429.22 & 28.2307 & 940.39 & 28.1806 & 3012.50 \\
\hline SY24 & 55 & 11.0 & 35.1029 & 374.78 & 34.9762 & 651.09 & 34.7975 & 1639.84 & 34.9462 & 3142.65 & 34.8172 & 9368.30 \\
\hline SY34 & 60 & 11.0 & 35.6893 & 435.39 & 35.5840 & 687.59 & 35.4590 & 1639.44 & 35.5567 & 3286.12 & 35.4618 & 8898.69 \\
\hline SY56 & 200 & 19.0 & 66.2883 & 19157.30 & 66.0273 & 42392.16 & 65.8655 & 152608.28 & 65.8893 & 217144.94 & 65.7343 & 446889.97 \\
\hline SY123 & 75 & 9.5 & 44.2845 & 324.84 & 44.2140 & 533.02 & 44.0380 & 1475.52 & 44.1984 & 2059.30 & 43.9885 & 5708.56 \\
\hline SY124 & 85 & 11.0 & 50.1517 & 598.98 & 49.9546 & 1031.78 & 49.7502 & 2505.45 & 49.8995 & 4403.21 & 49.6783 & 11484.14 \\
\hline SY134 & 90 & 11.0 & 50.7381 & 659.59 & 50.5624 & 1068.28 & 50.4117 & 2505.05 & 50.5100 & 4546.68 & 50.3229 & 11014.53 \\
\hline SY234 & 80 & 11.0 & 47.0353 & 473.48 & 46.8993 & 733.20 & 46.7088 & 1899.06 & 46.8457 & 3666.73 & 46.6912 & 10100.82 \\
\hline SY1234 & 110 & 11.0 & 62.0841 & 697.68 & 61.8777 & 1113.89 & 61.6615 & 2764.67 & 61.7990 & 4927.29 & 61.5523 & 12216.66 \\
\hline mean & & & 43.3582 & 1995.63 & 43.2235 & 4200.51 & 43.0525 & 14343.77 & 43.1668 & 20968.46 & 43.0075 & 44905.58 \\
\hline mean* & & & & 435.47 & & 728.55 & & 1774.27 & & 3134.23 & & 8361.54 \\
\hline
\end{tabular}

\subsection{Staged Packing}

In the simple decomposition method in $\S 5.4$, a clear line of demarcation exists between two sub-solutions. Actually, we have developed another staged scheme, where the overall packing is completed in stages, and the positions unused in the previous stage(s) can be still utilized in the following stage(s). Consequently, the area of the rectangle can be fully utilized and no demarcation line exists in the final packing configuration, though obtained in stages. Fig. 11 shows a three-stage packing for SY5 from decreasing orders of radii for each stage.

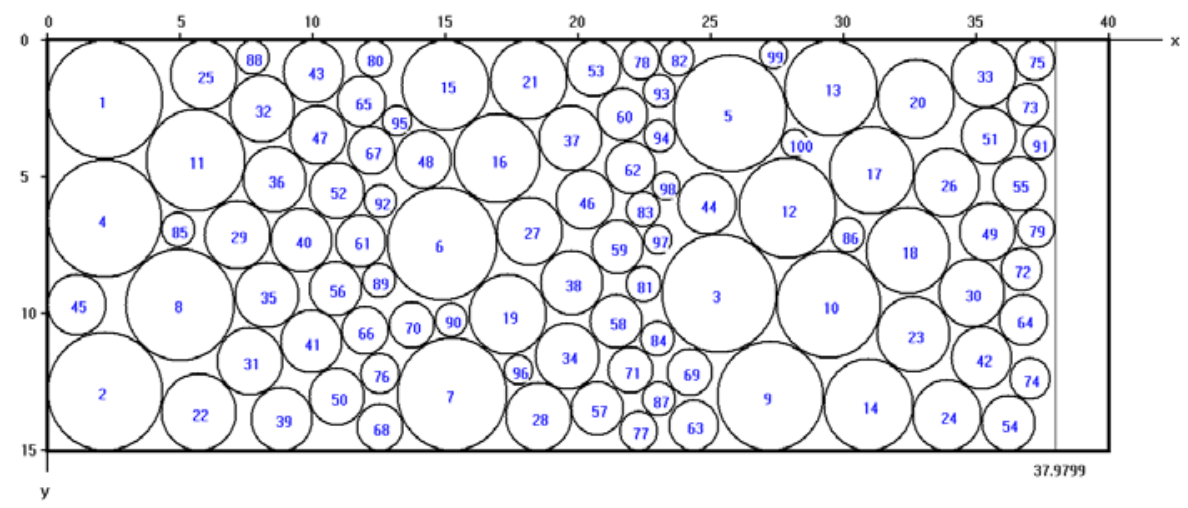

Fig. 11 A three-stage packing configuration for SY5 
In the staged scheme, the total circles to be packed are divided into several groups, ensuring the differentiation of circles selected in each group so that small circles can fill the gaps generated in packing large circles. The groups are then packed in respective stages. In each stage, IGA is still used to evolve packing solutions. Once the former-stage packing is completed, the three-type positions recorded are still kept for latter stages to utilize. Hence, a latter-stage circle maybe fits into a suitable position in former-stage packing. This evidently benefits the latter-stage packing. Consequently, the aggregate solution can be hopefully better than that from the above simple decomposition. Via such staged strategy, we not only take the advantage of decomposition to save solution time, but also enhance the overall solution quality.

\section{Conclusion}

For the challenging problem of packing non-identical circles into a rectangle with open length, this study first defined three types of positions possible to pack a circle, with which an arbitrary sequence of circles can be effectively changed into a feasible compact packing solution. Every time when a circle is packed at a chosen position, two type I positions are generated. In selecting a place for a circle among the unused type I positions, if an "infeasible” position is encountered, then a type II position is identified and recorded for latter selection. Similarly, if an infeasible type II position is encountered, then a type III position is discovered and recorded for latter selection. By this way, only a small number of positions of types II and III are recorded for selection, thus saving a lot of search time. With the novel position selection technique, a packing procedure is developed to convert a random circle sequence into a packing solution according to a given position selection criterion.

Secondly, diverse position selection criteria are tested, and it is found that the traditional criterion used in the literature is not the best one according to our numerical tests. An appropriate criterion selected in the packing procedure is one of the crucial factors for the proposed method to achieve new better solutions for some of the benchmark instances.

Thirdly, an improved genetic algorithm is proposed to search the solution space. Apart from the heuristic of the decreasing order of circle radii and the exchange-order tricks to quickly enhance the initial generation of solutions, a new operator (i.e. insert-mutation), inspired from the exchangeorder tricks, with the purpose of minor-adjusting a small part of the best solutions, is introduced into the classical genetic loop. Moreover, the investigated problem is indeed challenging for the genetic algorithm to explore the near-optimality, the unusual setting of low crossover rate and high mutation rate to overcome the defect of premature convergence is amazedly found more effective than simple increasing of the GA population.

Despite all these adaptations, the innate defect of the genetic algorithm, i.e., its search ability 
degrade quickly with the growth of the problem size, makes it dissatisfactory in solving large-size benchmarks. As such, simple decomposition is a direct compromise to take the advantage of the proposed algorithm for small-size instances; meanwhile, the idea of staged packing seems to be attractive and definitely worth further exploring.

It is noted that the investigated problem is indeed challenging for combinatorial optimization techniques, and the best solutions for the benchmarks are continually surmounted by researchers. This problem, as well as the benchmarks, can be used to test the search ability of dedicated algorithms. As for future work, decomposition, parallelization and more effective search algorithms would attract us to dedicate more research efforts on this problem. Exploration of similar packing problems, such as the packing of non-uniform circles in a circular container (which is still a challenging problem with many applications), would also deserve proper attention.

\section{Acknowledgement}

The authors are grateful to Stoyan and Yaskov, Hifi and M’Hallah, Huang and Li, Akeb and Hifi for the citation of their instances and results, and especially thank Dr. Yu Li and Dr. Hakim Akeb for providing us with their problem data. The authors would also like to thank the two anonymous reviewers for their constructive comments.

\section{References}

1. Akeb, H., Hifi, M.: Algorithms for the circular two-dimensional open dimension. International Transactions in Operational Research, 15, 685-704 (2008)

2. Akeb, H., Hifi, M., Negre, S.: An augmented beam search-based algorithm for the circular open dimension problem. Computers and Industrial Engineering, 61(2), 373-381 (2011)

3. Birgin, E.G., Martinez, J.M., Ronconi, D.P.: Optimizing the packing of cylinders into a rectangular container: a nonlinear approach. European Journal of Operational Research, 160, 19-33 (2005)

4. Castillo, I., Kampas, F.J., Pinter, J.D.: Solving circle packing problems by global optimization: Numerical results and industrial applications. European Journal of Operational Research, 191, 786-802 (2008)

5. Gen, M., Cheng, R.: Genetic Algorithms and Engineering Optimization. Wiley, New York, NY (1997)

6. George, J.A., George, J.M., Lamar, B.W.: Packing different-sized circles into a rectangular container. European Journal of Operational Research, 84, 693-712 (1995)

7. Goldberg, D.E.: Genetic Algorithms in Search, Optimization and Machine Learning. Reading, Addison-Wesley, MA (1989) 
8. Goldberg, D.E., Deb, K.: A comparative analysis of selection schemes used in genetic algorithms. In: Foundations of Genetic Algorithms, Rawlins, G. (eds), Morgan Kaufmann (1991).

9. He, Y., Wu, Y., de Souza, R.: A global search framework for practical three-dimensional packing with variable carton orientations. Computers \& Operations Research, 39(10), 2395-2414 (2012)

10. Hifi, M., M’Hallah, R.: Approximate algorithms for constrained circular cutting problems. Computers and Operations Research, 31, 675-694 (2004)

11. Hifi, M., Paschos, V.Th., Zissimopoulos, V.: A simulated annealing approach for the circular cutting problem. European Journal of Operational Research, 159, 430-448 (2004)

12. Huang, W.Q., Li, Y., Akeb, H., Li, C.M.: Greedy algorithms for packing unequal circles into a rectangular container. Journal of the Operational Research Society, 56, 539-548 (2005)

13. Kallrath, J.: Cutting circles and polygons from area-minimizing rectangles. Journal of Global Optimization, 43, 299-328 (2009)

14. Kubach, T., Bortfeldt, A., Gehring, H.: Parallel greedy algorithms for packing unequal circles into a strip or a rectangle. Central European Journal of Operations Research, 17, 461-477 (2009)

15. Poon, P.W., Carter, J.N.: Genetic algorithm crossover operators for ordering applications. Computers \& Operations Research, 22, 135-47 (1995)

16. Stoyan, Y.G., Yaskov, G.N.: Mathematical model and solution method of optimization problem of placement of rectangles and circles taking into account special constraints. International Transactions in Operational Research, 5, 45-57 (1998)

17. Stoyan, Y.G., Yaskov, G.N.: A mathematical model and a solution method for the problem of placing various-sized circles into a strip. European Journal of Operational Research, 156, 590-600 (2004)

18. Wu, Y., Li, W., Goh, M., de Souza, R.: Three dimensional bin packing problem with variable bin height. European Journal of Operational Research, 202, 347-355 (2010)

19. Yu, H., Zhang, L.: The augmented Lagrangian method for the packing of unequal circles within a strip. International Journal of Pure and Applied Mathematics, 18, 463-470 (2005) 


\section{Appendix: The Best Packing Layouts of SY1 to SY6 by IGA}

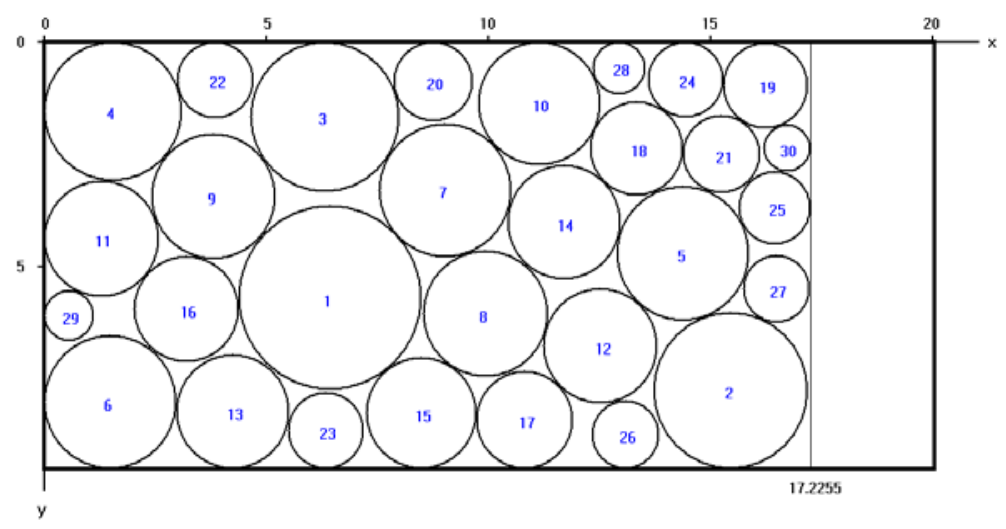

Fig. A1 The best packing of SY1 $(L=17.2225)$ obtained by IGA (popsize $\left.=2000, C_{r}: M_{r}=3: 7\right)$

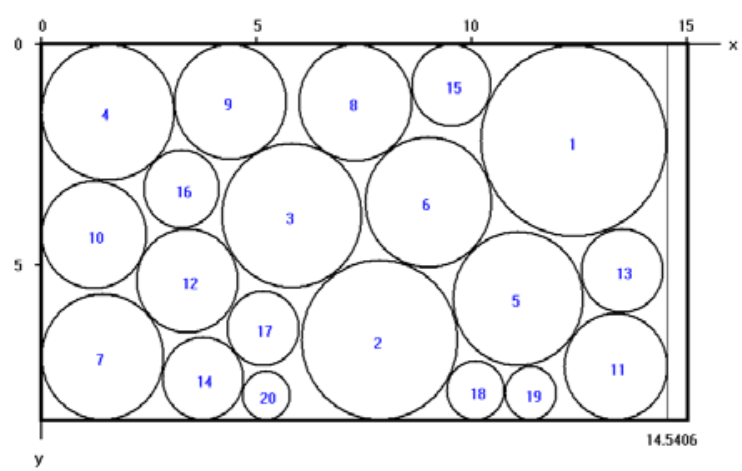

Fig. A2 The best packing of SY2 ( $L=14.5406)$ obtained by IGA (popsize $\left.=2000, C_{r}: M_{r}=3: 7\right)$

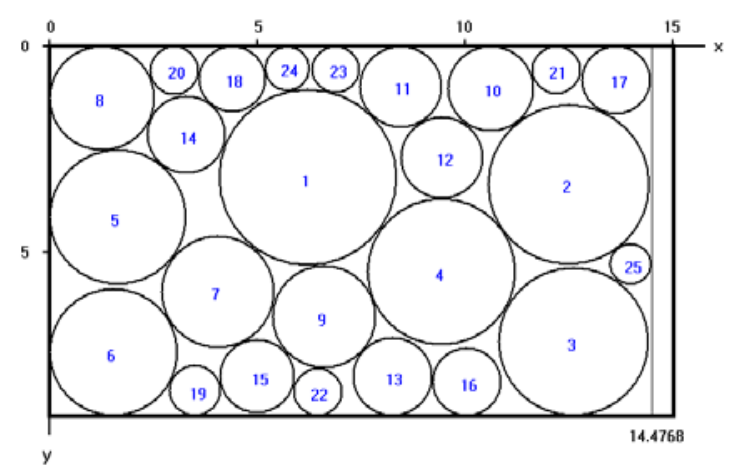

Fig. A3 The best packing of SY3 ( $L=14.4768)$ obtained by IGA (popsize $\left.=2000, C_{r}: M_{r}=3: 7\right)$

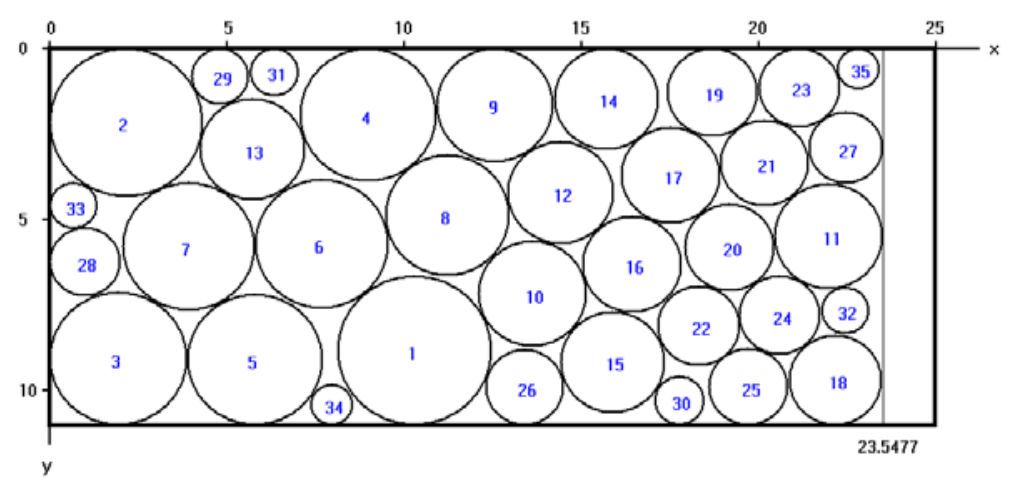

Fig. A4 The best packing of SY4 ( $L=23.5477)$ obtained by IGA (popsize $\left.=400, C_{r}: M_{r}=1: 9\right)$ 


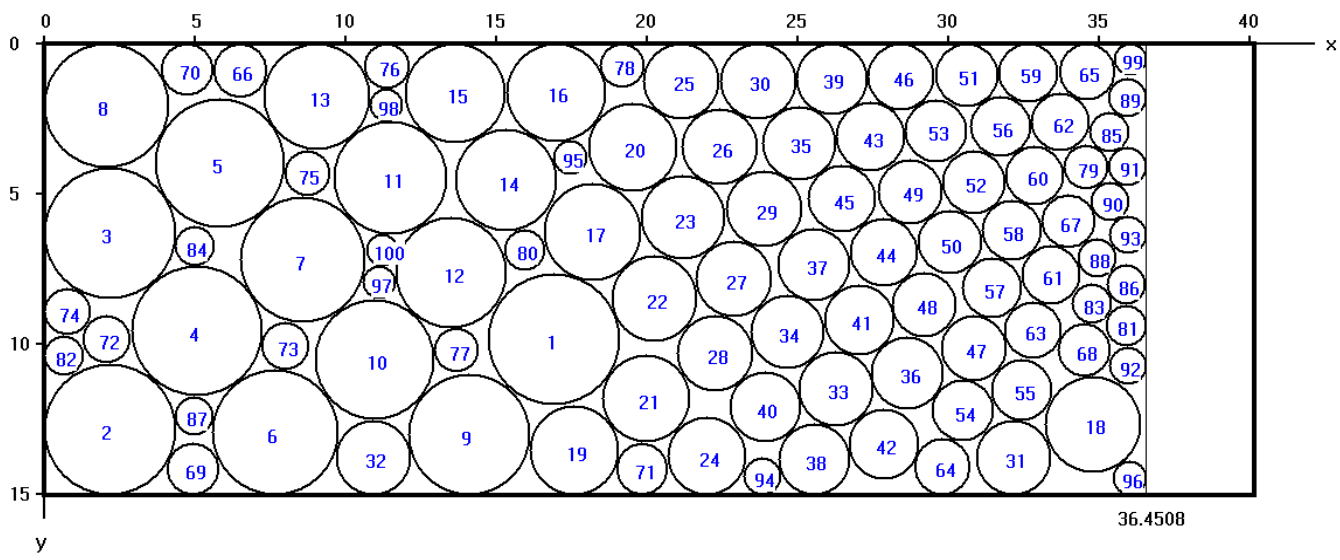

Fig. A5 The best packing of SY5 $(L=36.4508)$ obtained by IGA (popsize $\left.=400, C_{r}: M_{r}=1: 9\right)$

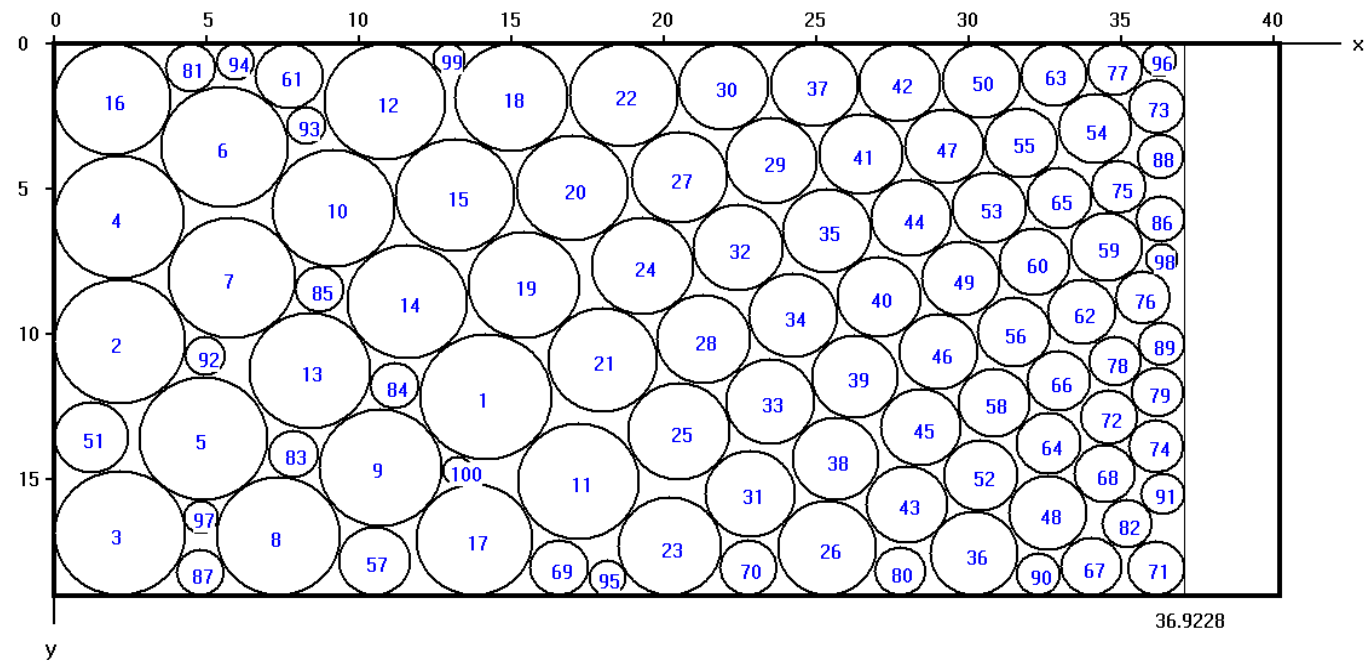

Fig. A6 The best packing of SY6 ( $L=36.9228)$ obtained by IGA (popsize $\left.=2000, C_{r}: M_{r}=3: 7\right)$ 